\title{
Supercapacitors: from the Leyden jar to electric busses
}

\author{
Deepak P. Dubal ${ }^{1} \cdot$ Y. P. $\mathrm{Wu}^{2} \cdot$ R. Holze $\mathrm{H}^{3}$
}

Received: 4 February 2016/Accepted: 19 April 2016/Published online: 17 June 2016

(C) Springer International Publishing Switzerland 2016

\begin{abstract}
Supercapacitors are introduced and reviewed as devices with very high electric power capability suggested to support and supplement other devices for electrochemical energy storage and conversion with higher energy content but lower power. They are currently of significant importance on the device and local (end-user) level already providing resources to meet power surge demands. In larger mobile systems (vehicles) they help meeting this demand and also offer high power storage capabilities when braking. On an even higher level, they can provide support for maintaining power quality and help in peak shaving. A brief historic background and general information is followed by an overview of materials and their combination into already marketed devices and in possible systems under development.
\end{abstract}

Keywords Supercapacitor - Energy storage - Energy conversion - Renewable energies - Power quality - Peak shaving $\cdot$ Load leveling

This article is an updated and extended version of a report to the members of the Deutsche Bunsen-Gesellschaft für Physikalische Chemie (Bunsen-Magazin 17 (2015) 216).

\section{R. Holze}

rudolf.holze@chemie.tu-chemnitz.de

1 Catalan Institute of Nanoscience and Nanotechnology, CIN2, ICN2 (CSIC-ICN), Campus UAB, 08193 Bellaterra, Barcelona, Spain

2 College of Energy, Nanjing Tech University, Nanjing 211816, Jiangsu, People's Republic of China

3 Technische Universität Chemnitz, Institut für Chemie, AG Elektrochemie, 09107 Chemnitz, Germany

\section{Introduction}

Electric energy is generally considered as being essential for industrialized societies and advanced economies. Hardly any part of daily life, industrial activity or social interaction, is conceivable without continuous, reliable and affordable supply of electricity. This applies with slightly different emphases to developing countries too. Providing potable water in sufficient quantities, enabling communication, supplying medical care and further basic functions are hardly possible without electricity. At places off the electric grid-and this is frequent in rural areas even in highly developed countries-where electric energy is obtained from renewable sources (wind, solar, hydro), storage of electric energy is needed to compensate the fluctuating supply. Integration of these sources into the electric grid is in addition a major option to move beyond the use of fossil fuels and nuclear energy. Because of the fluctuating power generation and consequently the mismatch between supply and demand storage of energy is essential as shown in a representative example in Fig. 1.

Another mismatch also suggesting the use of storage options is sometimes addressed as "peak shaving" PS, it designates the daily fluctuations of demand and their partial compensation on the grid level and is illustrated schematically in Fig. 2.

Basically the power generation capacity (i.e., the power supplied to the grid) has to match the maximum demand. In periods of lower demand, power stations are either running at reduced output or switched off, depending on the type of power station. Because most power stations, in particular thermal ones running on soft coal and on nuclear, respond very slowly to changing demand or are economically inefficient at reduced output, some excess power may still be offered in times of low demand. Sometimes this has 


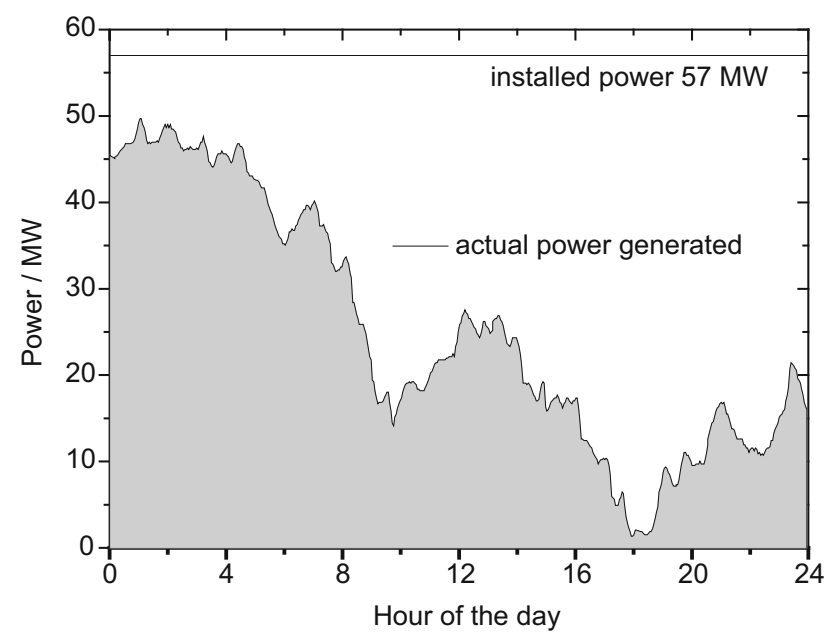

Fig. 1 Actual power delivery from a wind farm (76 turbines) in Chap-Chat, Quebec, Canada, on March 16th, 2004

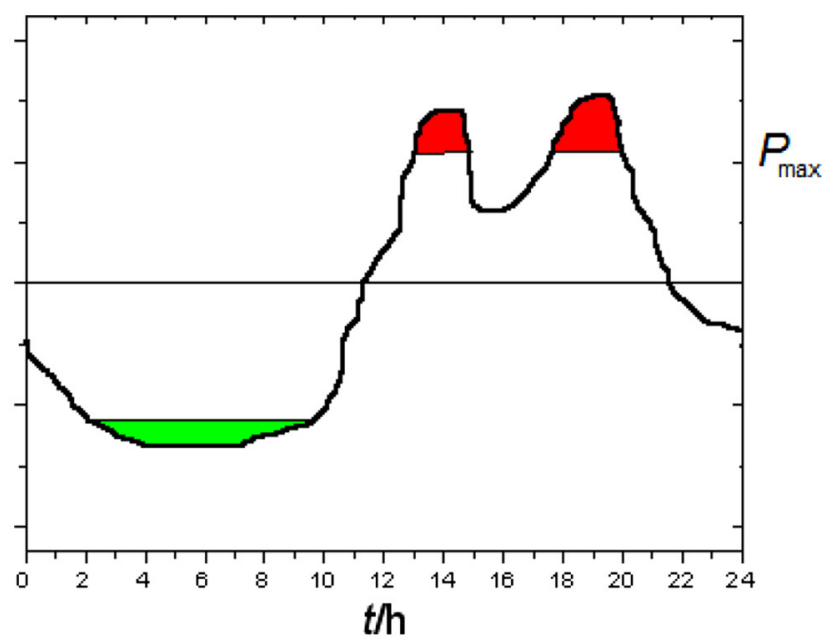

Fig. 2 Peak shaving, red energy supplied from storage by discharge; green energy received into storage by charge; the maximum power supplied into and demanded from the grid is labeled $P_{\max }$

been used to operate residential heating systems with thermal storage capability, it can also be used to replenish other devices for storing electric energy (see more below). Discharging these storage systems in times of high demand might reduce the required maximum power somewhat in PS consequently reducing the required total of installed generation capacity. An even better match would be achieved by load leveling LL as shown in Fig. 3.

The maximum power demand would be substantially lower, but the required storage capability is also vastly enlarged. Because the power generating systems would always be running at constant load, presumably at their best performance and efficiency, the overall balance in terms of losses, emissions and costs would be best-given the availability of affordable and reliable storage.

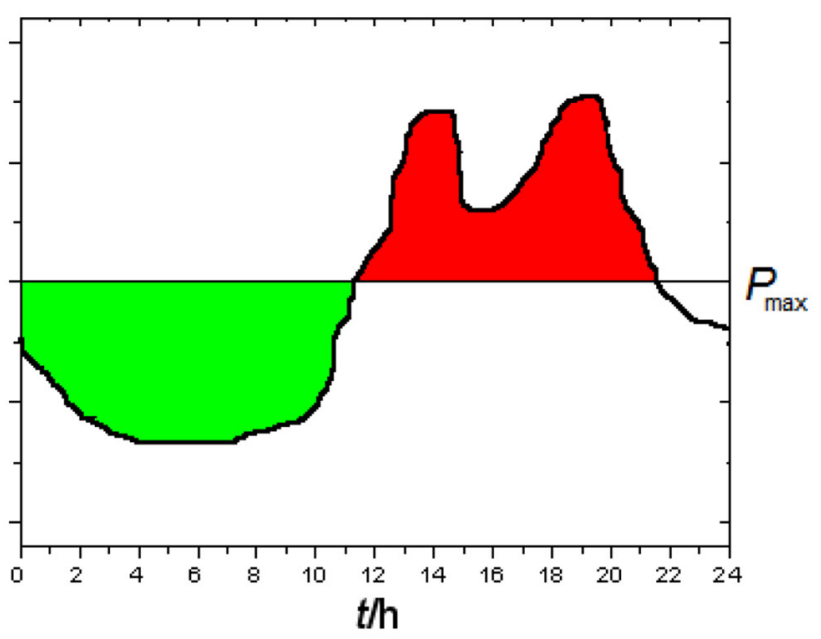

Fig. 3 Load leveling, red energy supplied from storage by discharge; green energy received into storage by charge; the maximum power supplied into and demanded from the grid is labeled $P_{\max }$

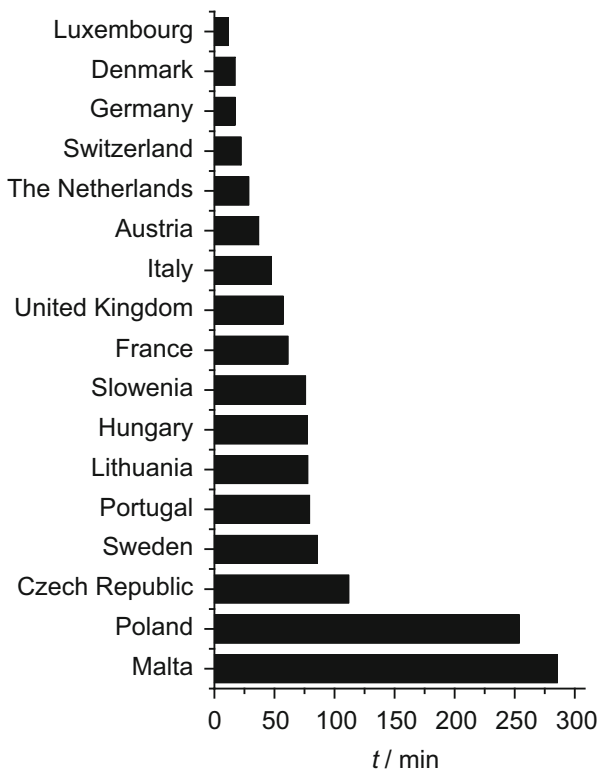

Fig. 4 Power outages in min year ${ }^{-1}$ in 2013 in Europe

For maintenance of grid stability (PQ: power quality, maintenance of frequency within fractions of one Hertz and voltage during sudden surges and drops of demand), storage facilities or electricity-generating power stations capable of rapid response are also required. The current status in Europe is mirrored in Fig. 4.

Elsewhere the situation is less satisfactory; large-scale blackouts in 2003 in the Northeast and in 2011 in the Southwest of the USA indicate substantial fragility even in highly industrialized economies.

The need for systems to store electric energy across a wide scale ranging from microbatteries for single devices 
at the end-user level to large assemblies supporting the grid of states and even larger entities has been addressed and reviewed repeatedly $[1,2]$.

A challenge worth mentioning beyond the scope of this contribution is efficient use of electric energy. On the device level significant progress has been achieved with many conceivable electric devices. In a broader sense, efficiency also includes balanced use of electric energy, i.e., matching supply and demand by switching off/on users depending on the available supply. This can be encouraged or even enforced by pricing schemes offering rebates at times of low demand from the grid and surcharges in times of high demand. Years back this was achieved in a most rudimentary way by counting (metering) devices operating with just two specific prices as a function of time of the day. More sophisticated devices ("smart counters/meters") provide much more refined options. This may include the integration of energy generation at the end-user level in particular by photovoltaics. Interconnecting these meters and providing more input from the electricity supplier as well as the consumer finally results in a "smart grid" [3]. Proper implementation might have results similar to the use of storage systems, but they will never replace them.

\section{Storing electric energy}

Electric energy can be stored directly, i.e., without any transformation step(s), in capacitors and inductances. Both options are utilized widely in electric and electronic devices ranging from large capacitors attached to electric motors [4] and huge transformers found in power plants to tiny inductances and blocking capacitances in mobile phones and microelectronics - to name just a few of many examples [5]. The underlying principles are hardly adaptable at least at first glance for large-scale storage of electric energy with amounts of energy and on timescales going beyond the extremely small and short ones effective in the mentioned applications. An inductance capable of storing just $216 \mathrm{kWh}$ at a current of $5700 \mathrm{~A}$ and a selfinductance of $48 \mathrm{Hy}$ - at a weight of 276 tons - is installed at CERN, Geneva, at the hydrogen bubble chamber. For larger storage projects (5000-10,000 MWh) according to estimates large coils (several $100 \mathrm{~m}$ in diameter) causing huge magnetic fields requiring remote or underground installation would be necessary. Equally disappointing results follow at first glance from similar considerations focusing on capacitances [6]. Nevertheless, both storage modes attract great interest because both are fundamentally capable of delivering the stored energy fast, at high rates (currents), i.e., at high power. The same applies to their capability to receive electric energy at high rates. Thus, capabilities are of substantial and growing interest for applications ranging from regenerative braking of vehicles (from small cars to railway trains) not connected to any grid or overhead wire to systems maintaining power quality in the electric grid of utilities. Although there is currently hardly any hope that inductive systems may become applicable capacitors have made huge progress moving way beyond the classic dielectric capacitor based essentially on the Leyden jar [7].

This overview starts with a brief look at the physical essentials, at the initial attempts to break the presumed boundaries and reviews the current state of devices (currently called supercapacitors in a rather diffusely defined way $^{1}$ ).

\section{The fundamentals}

Electric energy can be stored as electric charge $Q$ at a voltage $U$ in a capacitor of capacity (or capacitance, both terms are used almost synonymously despite the subtle physical differences ${ }^{2}$ ) $C$

$C=\frac{Q}{U}$.

The stored energy $W^{3}$ and the available power $P$ at constant $\Delta E$ (i.e., constant voltage $U$ ) are given by

$W=\frac{1}{2} C(\Delta E)^{2}=\frac{1}{2} C U^{2}$

\footnotetext{
${ }^{1}$ The mix-up related to naming will be considered below.

2 Terminology related to capacitors is apparently confused. A glance into textbooks of electric engineering and electronic circuit design suggests that capacitor is preferably used as the name of a passive component with capacitance as the name of its property, the storage capability, i.e. the "value" of the device in units of farad F, microfarad $\mu \mathrm{F}$, etc., but sometimes also the device itself. Practically as a synonym the term capacity is used to name the actual value of this property of a capacitor. Different from the terminology used with Ohmic resistors and confusing at first this is due to the fact that no "specific capacity" in the well-defined sense of the "specific resistance", i.e. the resistivity, exists. This is basically due to the fact, that capacitance contrary to resistance is no specific material property. Confusingly again frequently specific capacities or capacitances are reported. The provided numbers refer to the capacity per unit volume, unit weight or unit area. Correctly, the first should be called specific gravimetric, the second specific volumetric and the third area-specific (sometimes also areal) capacity. A similar confusion exists in the field of primary and secondary batteries. Instead of simply using the systematic and self-explaining terminology outlined above authors frequently attempt to enforce an almost personal terminology based on unsupported traditions and absent references.

${ }^{3}$ Unfortunately the symbol $E$ is used both for energy (being the equivalent of work $W$ ) and electric and electrode potential. To avoid confusion in the following equation $W$ is used for the former, $E$ for the latter-different from IUPAC conventions.
} 
$P=\frac{W}{t}$

$P=\frac{(\Delta E)^{2}}{\mathrm{ESR}}=\frac{U^{2}}{\mathrm{ESR}}$

with $\Delta E(=U)$ being the difference of electric (later also electrode) potentials between the plates (or electrodes) of the capacitor, $t$ the duration of discharge and ESR the equivalent series resistance, i.e., the electric series resistance ${ }^{4}$ of the device [8]. ESR is the sum of all Ohmic components inside a device between the external connectors (electronic/electrolytic resistance of wires, electrolyte solution, ${ }^{5}$ active masses, current collectors, etc.). Typical values of ESR are in the range of milliohms, actual values vary depending on construction of a capacitor and materials. The relationship $2 \mathrm{~b}$ between energy $W$, power $P$ and time $t$ is common. Because of the decreasing voltage during discharge associated with a decreasing current, the power also decreases. Equation $2 b$ takes into account such changes automatically. The relation between power, voltage and ESR is less obvious: power is equivalent to the product of voltage and current with the latter limited by voltage and internal (ESR) resistance $I=U /$ ESR. Thus, $P=U^{2} /$ ESR provides only a value at some time during discharge. Because mostly the maximum value of $U$ at the beginning of discharge is considered, the resulting power is the maximum value and it should be designated $P_{\max }$. Assuming a linear change of voltage during discharge and a final voltage of $U=0 \mathrm{~V}$, the average value will be $U / 2$, and the average current $I / 2$. Both values entered into the equation yield $P=U^{2} /(4 \cdot \mathrm{ESR})$. Because of obviously possible confusion with Eq. 2c it should be called $P_{\text {av }}$. The result should be the same as the result of $P=W / t$ (Eq. 2b). In supercapacitors employing materials showing superficial redox processes (generally called pseudocapacitive materials, for a discussion of terminology see below) neither voltage nor current decrease linearly with time (for details and examples see below), accordingly the results obtained with the latter equation may not be equivalent to $P=W / t$.

These relationships provide already some suggestions for research and development and connect options of employed materials and operation as discussed following.

\footnotetext{
${ }^{4}$ Sometimes the symbol $R_{\mathrm{S}}$ is used instead of ESR [8]. This use is discouraged, because it will result in confusion with the commonly established use of $R_{\mathrm{s}}$ for a serial resistance component in the display and handling of impedance data in equivalent circuits and for the solution resistance $R_{\mathrm{s}}$ in electrochemistry.

${ }^{5}$ Frequently electrolyte is used as a synonym of electrolyte solution. This obviously overlooks textbook knowledge; it causes confusion when taking into account molten salts and ionic liquids. Consequently, the correct terminology—although longer-is used in this text. In general considerations, electrolyte solution includes all ionically conducting phases (an even longer alternative term) between the active masses.
}

The capacity $C$ depends on the area $A$ of the plates of a capacitor, their distance $d$ and the dielectric constant $\varepsilon_{\mathrm{r}}$ of the material in between them according to

$C=\frac{\varepsilon_{\mathrm{r}} \varepsilon_{0}}{d} A$

with the distance $d$ between the plates of area $A$ with $\varepsilon_{0}$ the dielectric constant (of vacuum) and $\varepsilon_{\mathrm{r}}$ of any matter possibly in between the plates. An increase in capacitance will result in a corresponding increase in stored energy, an increase of operating voltage will have a relatively larger effect because of the quadratic term (Eq. 2a).

Early development aimed at increased surface areas and decreased distances can be envisaged when looking at the development from early dielectric capacitors to electrolytic capacitors and finally to electrochemical double-layer capacitors (EDLC).

The capacitance of the first device is given by the already mentioned mechanical dimensions and the dielectric properties of the insulating material between the electrodes. Very thin metal foils used as electrodes and equally thin foils of various (mostly polymeric) materials as insulators rolled up into cylindric or flat (after mechanical compression) capacitors or stacked up into prismatic ones are still the state of the art, for typical examples see Fig. 5.

Because of the limited electronic conductance of the used metal foils, interconnects, external connectors, etc., the device has an Ohmic resistance component different from the ideal zero value, and since all these contributions are connected in series the extended equivalent circuit as shown in Fig. 6 contains a resistive element ESR.

The required minimum thickness of the insulating material is given by its insulating properties (breakdown voltage); a practical limit is in many applications in lowvoltage electronic circuits given by mechanical handling properties of thin foils. Large capacitance values will thus result in capacitors with large mechanical dimensions. A further improvement can be gained using dielectric insulator materials with values of $\varepsilon_{\mathrm{r}}$ much larger than those of polymeric materials. Several inorganic oxides are popular candidates (e.g., $\mathrm{Ba}-\mathrm{TiO}_{3}$ ) [9]. Their mechanical properties require completely different processing technology again limiting the capacitance values and restricting the use of capacitors to cases where their particularly favorable highfrequency behavior is required and the sometimes very pronounced temperature dependency of $\varepsilon_{\mathrm{r}}$ is of no problem [10]. Further increase of surface area and decrease of distance is only possible employing a completely different approach. The true or real surface $\operatorname{area}^{6} A_{\mathrm{t}}$ of a sample (foil,

\footnotetext{
${ }^{6}$ This must not be confused with the electrochemically active surface area, which in most cases is much smaller than the true surface area implying an only incomplete utilization of available surface area.
} 
pellet, etc.) made by compressing a powder may be orders of magnitude larger than the geometric or apparent surface area $A_{\mathrm{g}}$ because of roughness or deliberately applied microstructuring; their relationship is expressed in electrochemistry frequently using a roughness factor $R_{\mathrm{f}}$ :

$A_{\mathrm{t}}=A_{\mathrm{g}} \cdot R_{\mathrm{f}}$.

Utilization of $A_{\mathrm{t}}$ is not possible with foil-shaped insulating materials, and even the other dielectrics mentioned above cannot be used economically on a large scale, i.e., for large capacitors. Instead an electrolyte solution is employed, which easily adapts to the rough, corrugated

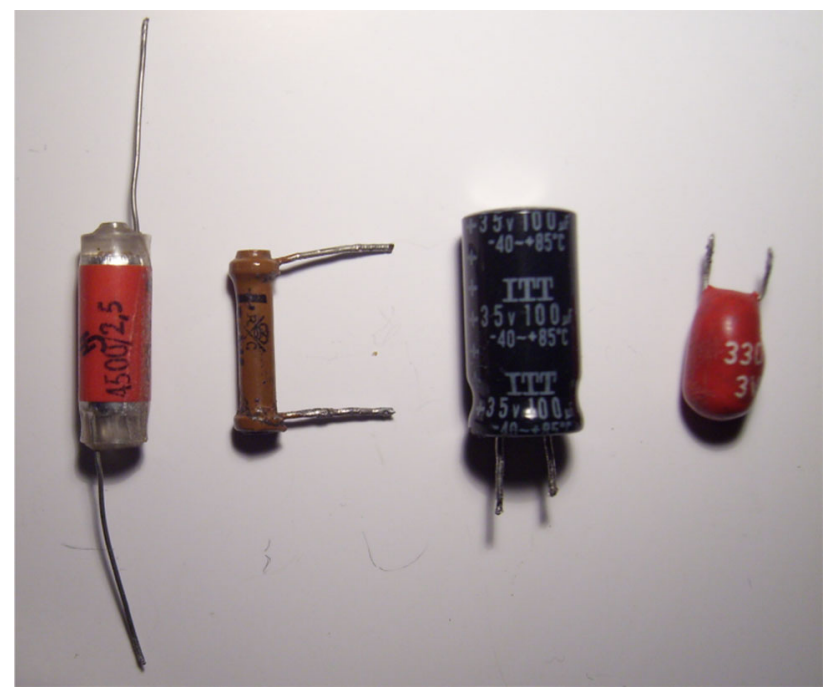

Fig. 5 Examples of dielectric capacitors with plastic foil dielectric, oxide insulator, electrolytic capacitor with aluminum and tantalum electrodes (left to right) surface. A second contact substituting the second electrode in a dielectric capacitor is needed, and it can be established easily by bringing another electronically conducting material with large true surface area (e.g., a roughened aluminum foil or a pellet of activated carbon with some binder) chemically stable towards the electrolyte solution into contact with it. The insulating dielectric material is prepared by forming it as a layer on the first electrode by oxidizing an aluminum foil surface previously roughened by a chemical or electrochemical process, or by oxidizing a porous body of tantalum powder. $\mathrm{Al}_{2} \mathrm{O}_{3}\left(\varepsilon_{\mathrm{r}}=7\right)$ is formed in the first case, $\mathrm{Ta}_{2} \mathrm{O}_{5}\left(\varepsilon_{\mathrm{r}}=27\right)$ in the second. Both oxides are excellent insulators even at very low thickness (thus enabling the small distance between the two phases: metal and electrolyte solution). The resulting device is a series connection of the dielectric capacitor just described and the double-layer capacitance established at the interface between the electrolyte solution and the second electrode. Because the double-layer capacitance $C_{\mathrm{dl}}$ is much larger than the dielectric one $C_{\text {diel }}$ the overall capacitance of the device is given by

$\frac{1}{C}=\frac{1}{C_{\mathrm{diel}}}+\frac{1}{C_{\mathrm{dl}}} \approx \frac{1}{C_{\mathrm{diel}}}$.

Further oxidation and associated growth of the dielectric layer at the aluminum or tantalum electrode can be avoided using this electrode as the negative pole in the application (polarized capacitor). The resulting device is shown schematically in Fig. 6b. Large capacitances again require large electrodes and/or thin dielectric layers limiting once more practically feasible storage devices. The inherently large interfacial capacitance of porous materials in contact
Fig. 6 Schematic cross sections, simplified and extended equivalent circuits of (a) dielectric capacitor, (b) electrolytic capacitor; (c) electrochemical double-layer capacitor. Inductive contributions/elements are not shown, they are of minor importance in most supercapacitor applications, $C_{\text {diel }}$ : capacitance established between two metallic electrodes separated by a dielectric medium, $C_{\mathrm{DL}}$ : capacitance of the electrochemical double layer, ESR: electric series resistance

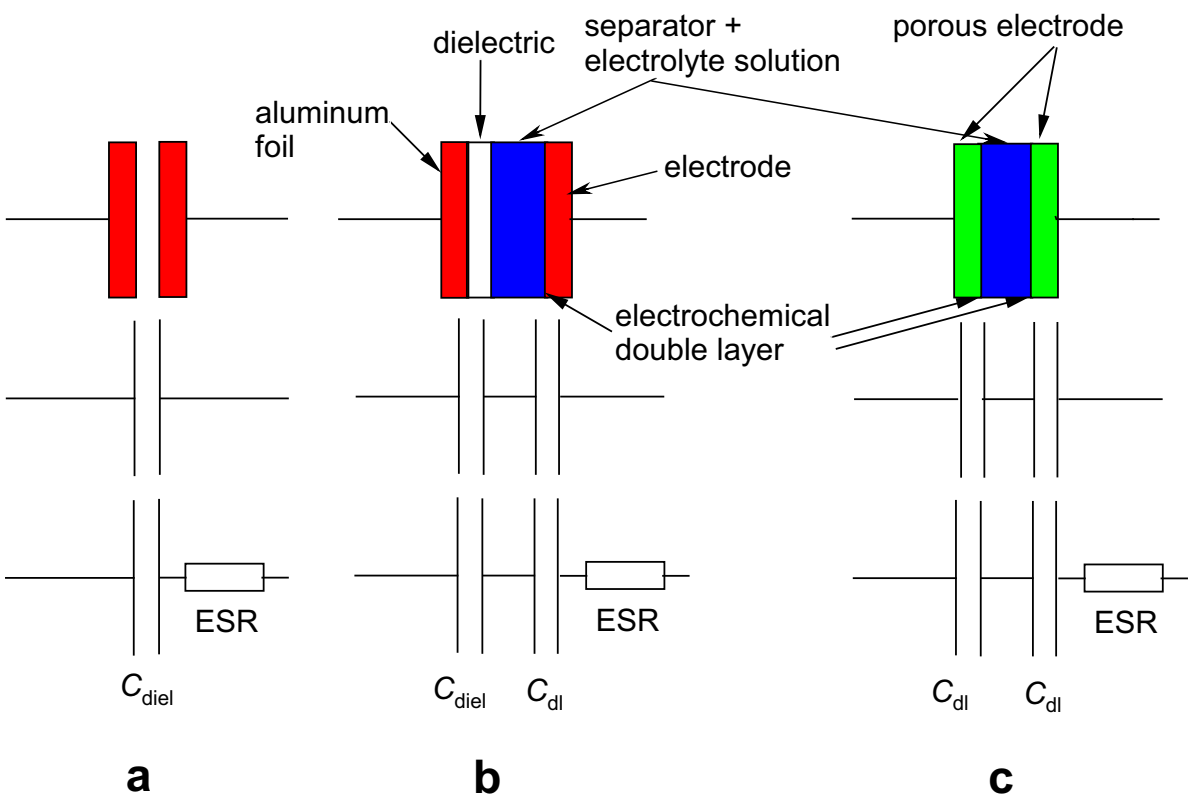


with electrolyte solutions already utilized to a rather small extent in the counter electrode of the electrolytic capacitor provided relief. The actual value of $C_{\mathrm{dl}}$ for an electrochemical interface between an electronically conducting phase (frequently and incorrectly called the electrode) and anionically conducting phase depends on several factors; a value of $C_{\mathrm{dl}} \approx 20 \mu \mathrm{F} \mathrm{cm} \mathrm{cm}^{-2}$ for an ideal smooth metallic surface in contact with an electrolyte solution is generally accepted. Taking porous materials, e.g., compressed pellets of activated carbons or carbon blacks with specific BETsurface areas of $2000 \mathrm{~m}^{2} \mathrm{~g}^{-1}$ (and more), large doublelayer capacitance values are conceivable [11]. This was already noticed in a patent [12] dealing with the electrochemical behavior of flooded porous electrodes. This discovery had no immediate consequences for capacitor development, thus the patent filed by Rightmire (SOHIO) [13] may be considered as the starting point of electrochemical double-layer capacitors [14]; although, even in 1977, the actual mode of storage was not fully understood as reported in a patent filing by Boos [15]. In this device, two identical porous electrodes with an electrolyte solution between them are assembled into a capacitor as schematically shown in Fig. 6c. Two double-layer capacitances are connected in series, the overall capacitance of the device is given by

$\frac{1}{C}=\frac{1}{C_{\mathrm{dl}}}+\frac{1}{C_{\mathrm{dl}}}=\frac{2}{C_{\mathrm{dl}}}$

Charge storage in the electrodes, actually at the interfaces, proceeds via localized accumulation of conductionband electrons in the electronically conducting phase and corresponding accumulation of counterbalancing ions in the ion-conducting phase. Energy storage is finally based on the potential difference between both electrodes with the potential being related to the charge difference between the electrodes. Possible capacitances are substantial, given sufficient conductance inside the device both in the ionically conducting electrolyte solution and the electronically conducting porous electrode is provided which is required for the movement of charges during charge/discharge of the device. Energy density is, nevertheless, still disappointing when compared with data for secondary batteries (see also Fig. 25).

As observed earlier in studies of numerous metals in contact with aqueous electrolyte solutions and named for the first time by Conway and Gileadi [16] a metal in contact with an electrolyte solution showing surface-confined redox processes (e.g., formation of a layer of surface oxide or hydroxide) with reaction products or intermediates (i.e., $\mathrm{OH}^{-}$in the example) strongly adsorbed (electrosorbed) at the metal surface shows a current response to a change of electrode potential very much like an interfacial capacitance, the authors called it redox

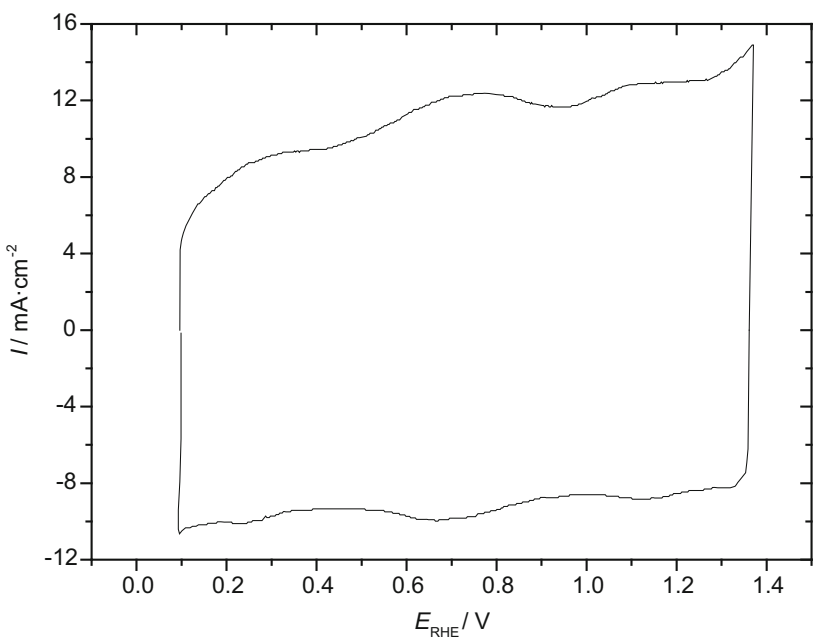

Fig. 7 Cyclic voltammogram (CV) of a $\mathrm{RuO}_{2}$ electrode in contact with an aqueous solution of $0.1 \mathrm{M} \mathrm{H}_{2} \mathrm{SO}_{4}$, vs a relative hydrogen electrode $E_{\mathrm{RHE}}, \mathrm{d} E / \mathrm{d} t=50 \mathrm{mV} \mathrm{s}{ }^{-1}$, nitrogen purged (based on data in [17])

pseudocapacitance ${ }^{7}$ [17]. Basically this term describes the current response of an electrode towards a changing electrode potential in cyclic voltammetry being more or less similar to the $\mathrm{CV}$ of a capacitor (compare Fig. 10 or 12 and Fig. 7 or 20 ):

$C=\mathrm{d} Q / \mathrm{d} E$

or rearranged

$I=C \cdot \mathrm{d} E / \mathrm{d} t$

The response being strikingly different from the response of a redox system of whatever kind employed in a battery electrode has been explained by considering the charge transfer reaction itself and in particular associated charge accumulation/depletion at the electrode/solution interface by strongly adsorbed reaction intermediates or products. A detailed study of the influence of adsorption isotherms and in particular strength of interaction between the adsorbed species (for details see [16]) as a function of electrode potential suggested current responses at particular conditions (coverage with adsorbed species and strength of interaction between them $)^{8}$ showing the mentioned capacitive shape. As a consequence no peaks are observed, instead a more or less constant current flows up to the

\footnotetext{
7 This term has been adopted rather strangely and lacking all logics into the name "pseudocapacitor" for a device incorporating electrodes showing this behavior. This aberration is not welcomed, its use is discouraged.

${ }^{8}$ In the abstract of Ref. [16] a possibly confusing or misleading reference to "activation energy" is made. This energy is mostly discussed with respect to the charge transfer reaction (see, e.g. derivation of the Butler-Volmer equation), but such influence would not cause the particular current response observed here.
} 
electrode potential where either potential change or current flow is stopped (fully charged/discharged state) or is reversed (change from charge to discharge mode or vice versa). Applicability of the formal relationship $\Delta Q=$ $C \cdot \Delta E$ (the stored charge $Q$ is a linear function of the associated change of electrode potential $E$ (voltage window $U$ for charge or discharge) with the capacitance $C$ being the proportionality factor) and the particular shape of the $\mathrm{CV}$ ("signature") and of the galvanostatic charge/discharge curve have been suggested as criteria to assign pseudocapacitive behavior [18]. Taking into account at first glance the surprising observation that some metal oxides show pseudocapacitive behavior and others do not, a closer look at metal oxide properties seems to be warranted. Some of the oxides are poor conductors or even semiconductors. Thus, valence changes of the metal ions and exchange of anionic species may affect the electronic interaction between metal ions ("electronic communication" as suggested by Robin and Day [19]) in the solid and subsequently the redox behavior also resulting in the absence of visible peaks. Instead, a superposition of numerous peaks caused by redox processes with only slightly different redox potentials (the slight shifts being caused by said communication) results in a more or less constant current.

Details of metal oxide properties, values of band gaps and the relative position of band edges with respect to electrochemically accessible electrode potentials (within the electrochemically possible potential window fixed by electrolyte solution decomposition) have been inspected closer by Young et al. taking $\mathrm{MnO}_{2}$ as a particularly fascinating example [20]. Considering electrochemically accessible states in the metal oxide it turns out that with $\beta$ $\mathrm{MnO}_{2}$ these states are all within the conduction band, no charge transfer is possible. This already explains the very small specific capacitance of this material. The much larger values for $\alpha-\mathrm{MnO}_{2}$ are in part due to the much better match between states within the band gap which may switch charge and thus contribute to storage capacity and states in solution within the available electrochemical window. Further structural details of the various crystallographic phases, film thickness, $\mathrm{pH}$, etc., support the proposed model of charge storage going way beyond the earlier suggested simple explanation based on tunnel sizes inside the metal oxide structure.

In Fig. 7 a typical $\mathrm{CV}$ is shown with the redox reaction $\mathrm{RuO}_{2-\delta}(\mathrm{OH})_{\delta} \rightleftarrows \mathrm{RuO}_{2}+\delta \mathrm{e}^{-}+\delta \mathrm{H}^{+}$with $0 \leq \delta \leq 1$

Reasons for this shape, in particular for the frequently observed absence of well-defined current peaks (only weak waves on top of huge currents are seen in Fig. 7) depend on the identity of the material and will be discussed below.
Utilizing this phenomenon at metal or metal compound surfaces with large specific surface areas in contact with electrolyte solutions, pseudocapacitances 10 to 100 times of simple double-layer capacitances with respect to the real electrode surface can be obtained [21]. The discovery of the redox behavior of intrinsically conducting polymers and the search for possible applications initiated thereby has initiated intense research aiming in to the same direction: utilizing redox processes in particular at the solid surface, i.e., at the polymer/solution interface. Although they are basically behaving like a pseudocapacitive material they are treated separately below because of the fundamentally different mechanisms of operation.

\section{Experimental aspects}

Before inspecting the various materials suggested as active masses for supercapacitors, a brief look at the measurement of the presumably most important materials property, its storage capability, is helpful. Confusion, poorly defined conditions and subsequently rather incompatible and thus incomparable data are presently the result. Since the race goes towards ever higher capacitance values and longer lifetimes a definition of measurement conditions is required, some attempts at this with only limited success so far have been reported [22, 23]. A brief overview of the basics follows.

Given the definition of Eq. 1, measurement of the capacitance value is simple: a record of the amount of transferred charge $Q$ (i.e., integrated flow of current over charging time) associated with an increase of the voltage applied to the terminals of a capacitor (or vice versa) will be enough. In case of a step-wise change of applied voltage, the initially large current (limited only by the Ohmic resistance of the employed electric circuit of the power supply and further limitations of the associated electronic circuits) providing charging at constant voltage might cause difficulties in determining the requested integral. A typical result illustrating this problem is shown in Fig. 8.

Current limitation in the supply sets in early, the rise is far from ideal. The current decay does not fit expectations for loading of a capacitor (with or without an internal Ohmic resistance, for a respective result see Fig. 9). No evaluation is attempted. Given the relationship between the flowing current $I$ and the applied voltage $U$, the described experiment results in a poorly defined situation:

$I=\frac{U}{R} e^{-\frac{t}{R C}}$.

The resistance $R$ in the equation is not defined: the actual value of ESR (even though it may be small) is not 


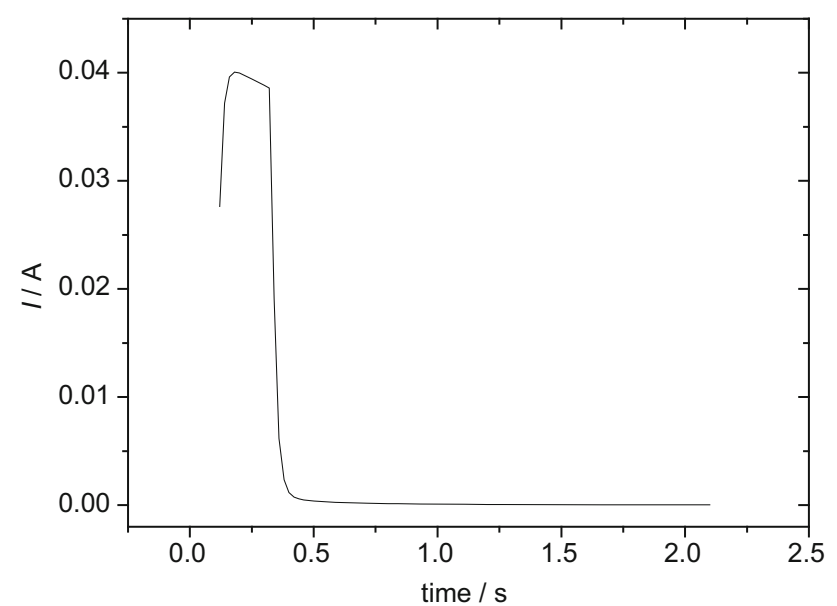

Fig. 8 Current vs. time plot recorded with an electrolytic capacitor of nominally $2200 \mu \mathrm{F}$ capacitance

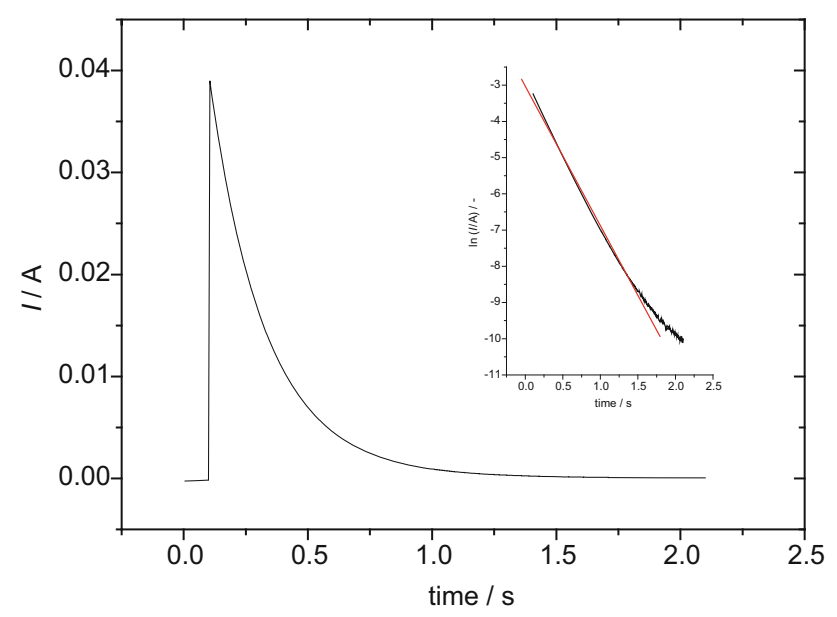

Fig. 9 Current vs. time plot recorded with an electrolytic capacitor of nominally $2200 \mu \mathrm{F}$ capacitance in series with an Ohmic resistor of $100 \Omega$; inset semilogarithmic plot

known, no further resistor has been included. The same experiment with a properly defined resistor $R=100 \Omega$ (certainly much larger than the ESR) yields a different result (see Fig. 9).

Evaluation is possible by integration or by taking the slope of the semilogarithmic plot (see inset) according to

$\ln I=\ln \left(\frac{U}{R}\right)-\frac{t}{R \cdot C}$

rearranged and differentiated

$\frac{\partial \ln I}{\partial t}=-\frac{1}{R \cdot C}$

yielding again the nominal value of the measured capacitor given the knowledge of the value of $R$.

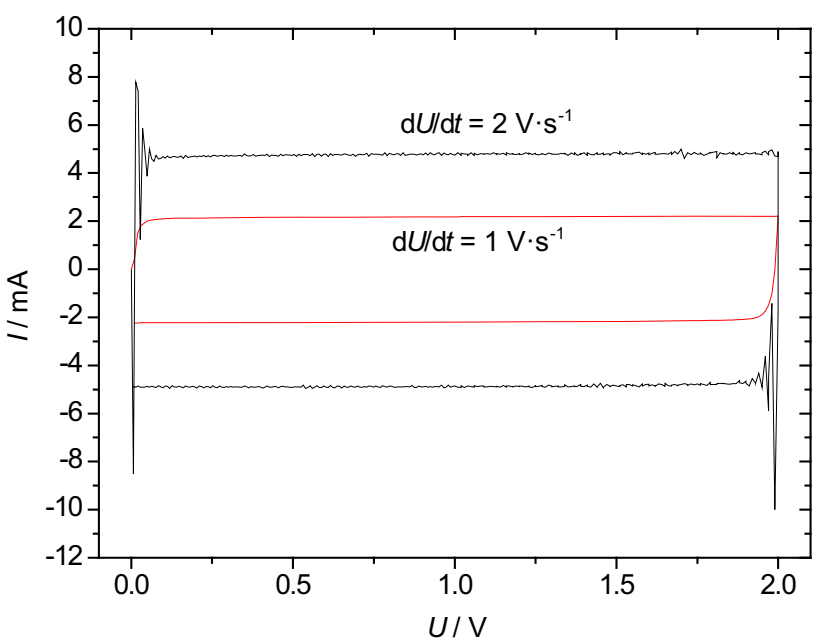

Fig. 10 Current vs. voltage plots at different scan rates (as indicated) recorded with an electrolytic capacitor of nominally $2200 \mu \mathrm{F}$ capacitance

A constant change of voltage is an option avoiding the apparent problems associated with sudden voltage changes. A typical result is shown in Fig. 10.

Although the used electrolytic capacitor is not an ideal capacitor at all the behaviors fits the expectation: at a constant value of $C$ and a constant change of voltage $\mathrm{d} U /$ $\mathrm{d} t$ the current $I$ is also constant. Obviously the current value $I$ and the rate of voltage change would be enough to calculate the capacitance-more on this below. At points of scan direction reversal, the potentiostat used in this experiment started to oscillate, the charging current can be read easily, nevertheless, yielding for both scan rates a capacitance of $2300 \mu \mathrm{F}$ well within the tolerance of the component.

The second approach utilizing a constant charging current avoids the previously mentioned problems and is used more frequently (galvanostatic charge/discharge curves) yielding a typical result as shown in Fig. 11.

Again the behavior fits the expectations: a strictly linear shape implying pure capacitive behavior. A capacitance of $2100 \mu \mathrm{F}$ can be calculated. In the electronic laboratory, frequently methods and devices using alternating current determining the apparent capacitive resistance or circuits automatically evaluating charging curves are employed.

In studies of electrochemical capacitors, measurements of both the capacitance of a single interface at the investigated (working) electrode and of the complete capacitor assembled with two electrodes are possible, the former are the most frequently encountered ones. The differential double-layer capacitance $C_{\text {diff }}$ is given as 


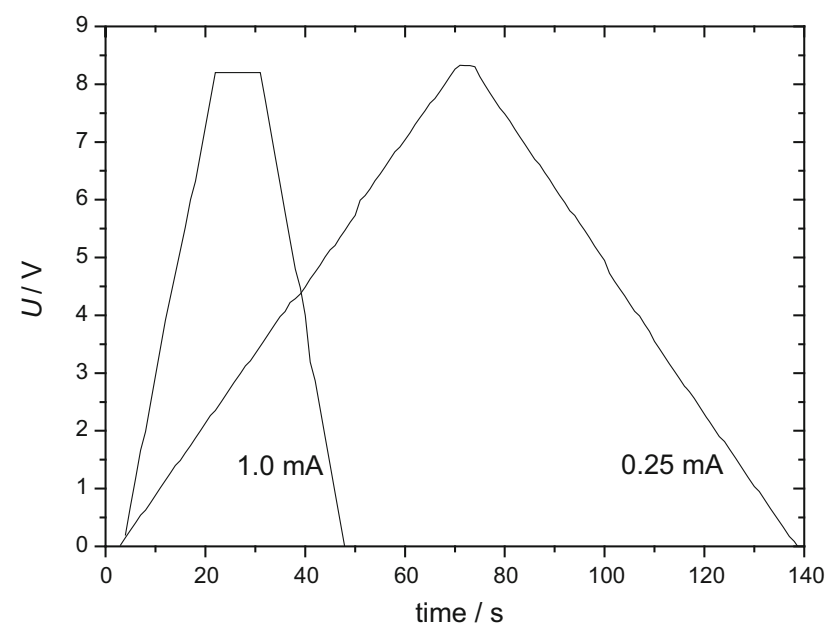

Fig. 11 Voltage vs. time plots of charge/discharge at various constant currents into an electrolytic capacitor of nominally $2200 \mu \mathrm{F}$ capacitance

$C_{\text {diff }}=\frac{\mathrm{d} Q}{\mathrm{~d} E}$

with the charge $Q$ and the electrode potential $E$. The integral double-layer capacitance $C_{\text {int }}$ (less frequently used) refers to the charge $Q$ associated with a given change of electrode potential from the electrode potential of zero charge $E_{\mathrm{pzc}}$ (or another point of reference) to a potential $E$ :

$C_{\text {int }}=\frac{Q}{E-E_{\mathrm{pzc}}}$

Inspection of a typical current response of an electrochemical interface (an electronically conducting phase in contact with an ionically conducting one) of a platinum sheet in contact with an aqueous solution of sulphuric acid in an electrode potential range where no Faradaic reaction proceeds provides suggestions for measurements as shown in Fig. 12.

In electrochemical studies with cyclic voltammetry $\mathrm{CV}$ the working electrode potential is changed linearly with time at a scan rate $\mathrm{d} E / \mathrm{d} t$ between a positive limit $E_{\mathrm{a}}$ and a negative one $E_{\mathrm{c}}$. An electrochemical double layer established at the interface between the electronically and the ionically conducting phases with a constant, i.e., electrode potential-independent interfacial capacitance, will yield an almost constant current as shown above in Fig. 12 (compare with Fig. 10). The slight tilt is due to the Ohmic potential drop across the electrolyte solution and the onset of Faradic reactions. As expected the current increases with growing electrode potential scan rate. A plot of current read at $E_{\mathrm{RHE}}=550 \mathrm{mV}$ vs. scan rate (Fig. 13) yields a straight line further confirming the capacitive (non-faradaic) nature of the current, from the slope the capacitance

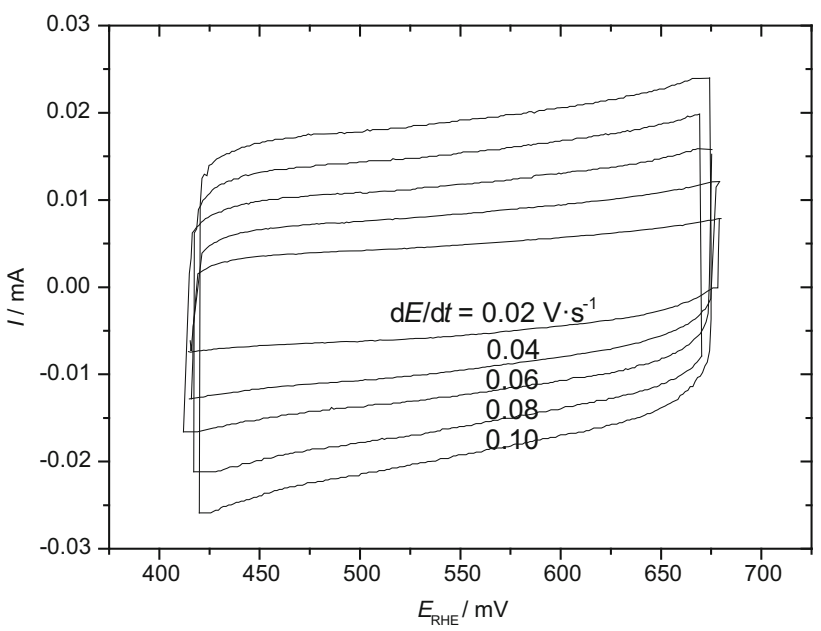

Fig. $12 \mathrm{CVs}$ of a platinum electrode in contact with an aqueous electrolyte solution of $0.05 \mathrm{M} \mathrm{H}_{2} \mathrm{SO}_{4}$ vs. a relative hydrogen electrode $\left(E_{\mathrm{RHE}}\right)$ in the double-layer region, nitrogen purged

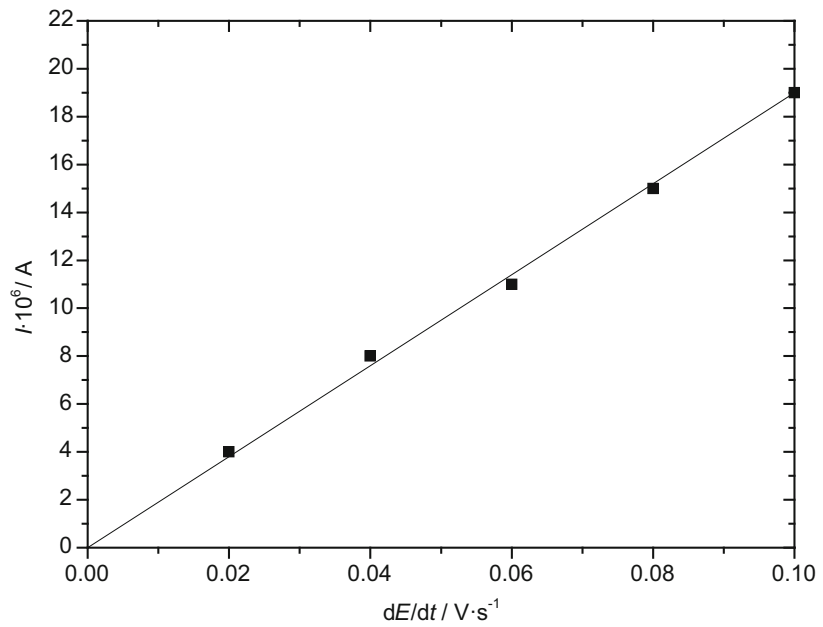

Fig. 13 Plot of current at $E_{\mathrm{RHE}}=550 \mathrm{mV}$ in the double-layer region as a function of scan rate for a platinum electrode of $3 \mathrm{~cm}^{-2}$ geometric surface area in an aqueous electrolyte solution of $0.05 \mathrm{M}$ $\mathrm{H}_{2} \mathrm{SO}_{4}$, nitrogen purged

value $C_{\text {diff }}=194 \mu \mathrm{F}$ can be read immediately. The current values are of course approximately average ones.

As an alternative, helpful in particular, when the current in the studied electrode potential window varies (showing a peak) integration of the current over electrode potential is possible as shown in Fig. 14 for a dataset from Fig. 13. This is basically equivalent to determining the average current and applying the previously used procedure. The obtained area (in units of VA) is divided by the applied scan rate and the potential difference between anodic and cathodic limit yielding again the capacitance $C_{\mathrm{int}}=192 \mu \mathrm{F}$. 


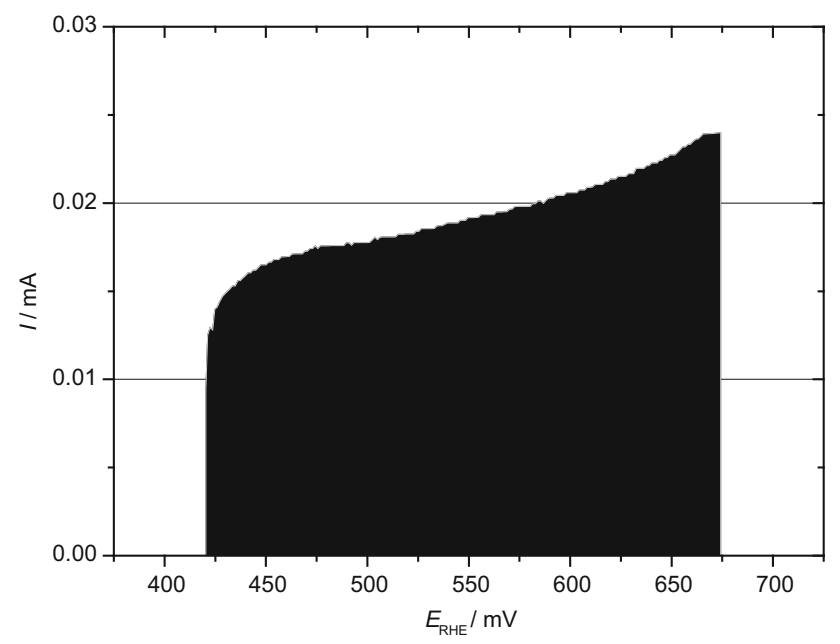

Fig. 14 Evaluation of positive going scan from $\mathrm{CV}$ in Fig. 12 at $\mathrm{d} E /$ $\mathrm{d} t=0.1 \mathrm{~V} \mathrm{~s}^{-1}$

CVs of supercapacitor electrodes may look different (see Fig. 15). Rarely-even with carbon-based electrodes - a shape of the CV closely resembling that shown in Fig. 12 is observed, thus taking the current at a single electrode potential will most likely yield useless results. In case of pseudocapacitive electrode materials showing more or less well-defined current peaks, the peak value must not be used because it will result in completely meaningless (over-blown) capacitance values. Instead, the integrated value of the part of the $\mathrm{CV}$ recorded in positive or negative going electrode potential direction with (anodic and cathodic) potential limits $E_{\mathrm{a}}$ and $E_{\mathrm{c}}$ should be used (not half of the correctly integrated total area-because done properly zero should be the obviously useless result when positive and negative charges cancel each other)

$C=\frac{\int_{E_{\mathrm{c}}}^{E_{\mathrm{a}}} I \mathrm{~d} E}{\Delta E \cdot \frac{\mathrm{d} E}{\mathrm{~d} t}}$.

Specific values of gravimetric capacitance $C_{\mathrm{g}}$ can be obtained by dividing by the actual mass $m$ of active material or of the electrode (including masses of binder and conducting additives, but almost never the current collector):

$C_{g}=\frac{\int_{E_{\mathrm{c}}}^{E_{\mathrm{a}}} I \mathrm{~d} E}{m \cdot \Delta E \cdot \frac{\mathrm{d} E}{\mathrm{~d} t} .}$

Values with respect to volume $V$ or apparent surface area $A$ are obtained by dividing with $V$ or $A$, respectively.

Capacitances can be calculated also from galvanostatic charge/discharge curves for time intervals from beginning $t_{\mathrm{i}}$ to end $t_{\mathrm{f}}$, for an example see Fig. 16. In addition to current densities defined with respect to the geometric electrode surface area (the most frequent standard interpretation of

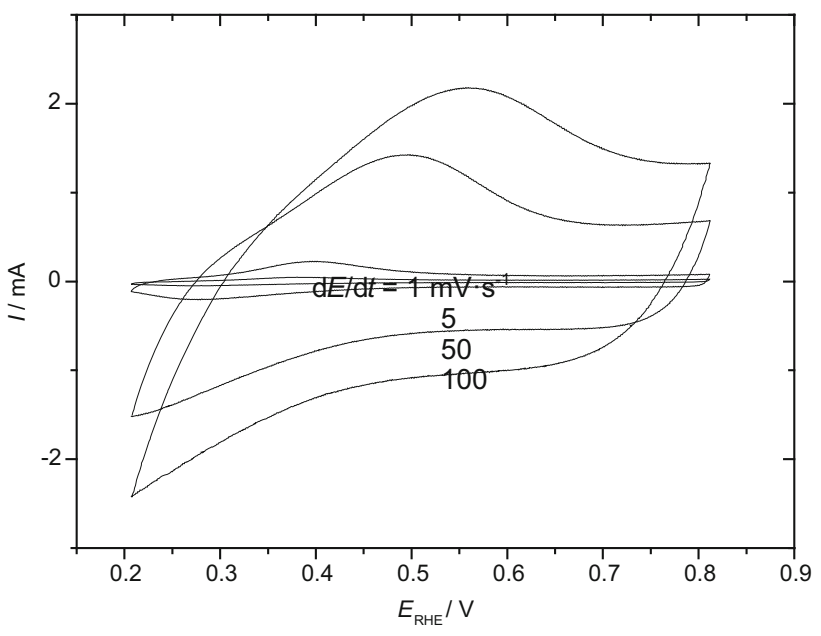

Fig. $15 \mathrm{CV}$ of a polyaniline-coated stainless steel grid electrode $\left(1 \mathrm{~cm}^{2}\right)$ in contact with an aqueous electrolyte solution of $1 \mathrm{M}$ $\mathrm{HClO}_{4}$, at different scan rates, nitrogen purged

current density) currents are given with respect to the mass of electrode material $(A / g)$. Because rarely the curves obtained with pseudocapacitive materials are exactly straight lines-in this case the slope would be sufficient for further calculation-integration again is needed:

$C_{\mathrm{g}}=\frac{I \cdot \int_{t_{\mathrm{i}}}^{t_{\mathrm{f}}} E \mathrm{~d} t}{(\Delta E)^{2} \cdot m}$.

Although there is apparently no established and generally accepted procedure (or even standard) to determine Coulombic (charge) storage capability of electrode materials (the terms capacitance or capacity are avoided here to minimize the risk of confusion), suggestions have been reported [24]; a particularly impressive example of associated pitfalls has been discussed [25].

\section{Materials and devices}

The two classes of electrode materials and devices made with them are described in detail, further extended overviews are available $[22,26-32] . \mathrm{SCs}^{9}$ wherein both

\footnotetext{
$\overline{9}$ The terms supercapacitor ${ }^{\mathrm{TM}}$ (as well as ultracap/ultracapacitor) or abbreviated supercap (SC) seemingly lack a generally accepted, proper definition. At first glance it appears sufficient to assume that capacitors based on the capacitive properties of the electrochemical double layer instead of a dielectric material like $\mathrm{Al}_{2} \mathrm{O}_{3}$ or $\mathrm{Ta}_{2} \mathrm{O}_{5}$ showing huge capacities are correctly called supercapacitors. Temporarily, the latter term was trademarked (from August 1978 on) to NEC Corporation; currently this protection has apparently expired. The acronym SC seems to be too short to enable immediate identification. Acronyms like ES for electrochemical supercapacitor or FS for Faradaic supercapacitor do nothing beyond enlarging the confusion. Recently, this device wherein purely electrostatic charge storage in the double layer is operative has been frequently called
} 
Fig. 16 Charge/discharge galvanostatically at various $\left(\mathrm{A} \mathrm{g}^{-1}\right)$ with $\mathrm{Cu}_{2} \mathrm{O}$ in an aqueous solution of $0.5 \mathrm{M}$ $\mathrm{K}_{2} \mathrm{CO}_{3}$ (unpublished data provided by $\mathrm{Yu} \mathrm{Liu)}$ curves obtained constant current densities

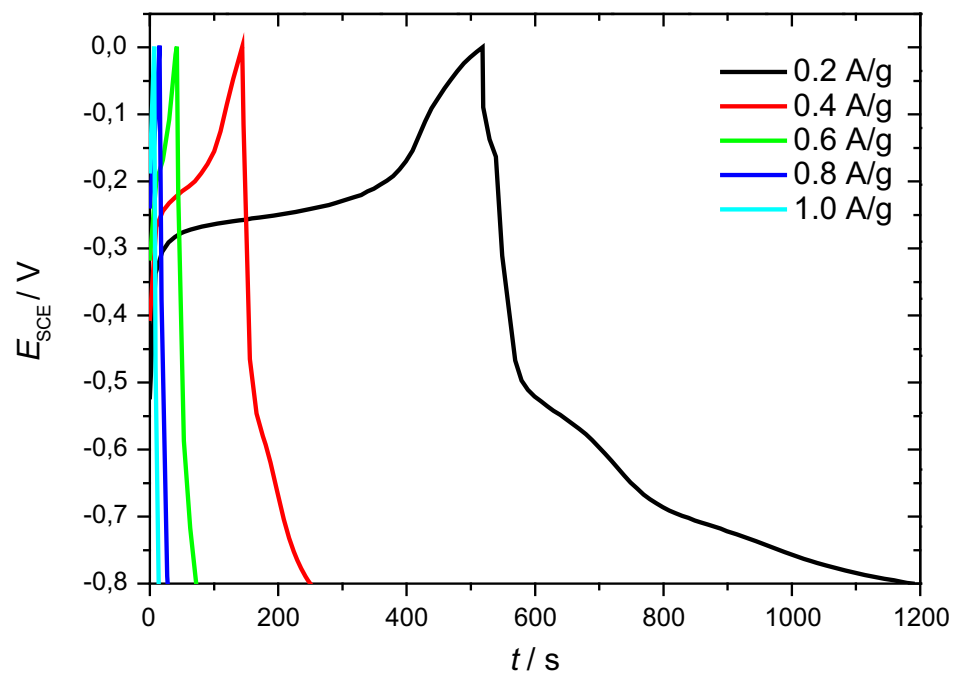

Capacitive materials

electrodes belonging to one of the classes of materials discussed below are identical are generally called symmetric, those combining electrodes from two classes (with the pseudocapacitive electrodes sometimes called battery electrodes) are called asymmetric. The term hybrid is also applied liberally; mostly it is used to name electrodes wherein materials of two classes are combined, i.e., a capacitive and a pseudocapacitive material. Depending on the chemical composition of the material and the assumed or even proven mode and intensity of interaction between the constituents, the term composite is also applied sometimes, it is presumably more appropriate in most cases. This terminology should not be confused with the concept of "hybrid energy storage systems", which describes a combination of various devices, e.g., a supercapacitor and a fuel cell or a lithium-ion battery, with the aim of providing both high power and high energy [33-35].

\section{Footnote 9 continued}

EDLC (electrochemical double-layer capacitor). Thus, it appears to be reasonable to call devices, wherein charge storage is based both on electrostatic charge separation (like in an EDLC) and on Faradaic redox processes (pseudocapacity) supercapacitors. Because of the combination of these fundamentally different charge storage mechanisms, these devices are also sometimes called hybrids-adding further to the confusion. In the present report, supercapacitors are such hybrid devices, the term ultracapacitor is not used at all. Its use to designate only those devices employing pseudocapacitances seems to be a loosing proposition [A. Burke, J. Power Sources 91, 37 (2000).]. The statement that Conway coined the term supercapacitor in 1991 is apparently erroneous. The rich collection of terms-some of them presumably protected by trademarks-does not help really: APowerCap, BestCap, BoostCap, CAP-XX, DLCAP, EneCapTen, EVerCAP, DynaCap, Faradcap, GreenCap, Goldcap, HY-CAP, Kapton capacitor, Super capacitor, SuperCap, PAS Capacitor, PowerStor, PseudoCap, etc.
Many active materials-whether they behave purely capacitive or pseudocapacitive ${ }^{10}$ - are electronically conducting (e.g., activated carbon, $\mathrm{RuO}_{2}$ ) with the conduction being supported by various mechanisms. Many materials are poor conductors or semiconductors, and some materials change their conductance as a function of the state of charge (a particularly impressive example is an intrinsically conducting polymer with changes of many orders of magnitude). To sustain the flow of current during operation, active masses are mixed with electronically conducting additives, most frequently from carbons ranging from acetylene black to graphene, carbon nanotubes, etc. Because these additives contribute barely to the storage capacity they will decrease the specific storage capacity of active materials. Quite obviously this suggests addition of only the minimum necessary to perform the required duty. Instead additions are either determined by trial-and-error (the exemption) or by tradition, i.e., staying with a number once established somehow (the rule).

\section{Electrochemical double-layer capacitors}

These devices (the acronym EDLC appears to be again subject of personal variations sometimes) employing in a symmetric arrangement two mostly carbon-based

\footnotetext{
${ }^{10}$ The term pseudocapacitive should be applied correctly only to those electrode materials showing a capacitive response in cyclic voltammetry or in galvanostatic charge/discharge experiments as discussed above. In the overwhelming majority, the term is used in a less precise and wider meaning and applied to all materials showing Faradaic response (like a battery electrode) and of course relatively smaller but omnipresent capacitive contributions from the electrochemical double layer. The latter meaning and terminology is pursued in this report.
} 
electrodes are the most frequently encountered supercapacitors at the time of writing this overview [11]. In particular, activated carbons (ACs) are well known for their huge internal surface areas as determined by the BrunauerEmmett-Teller (BET) method [36, 37]. As indicated in the introduction, pellets made from AC brought into contact with an electrolyte solution yielded first evidence of large double-layer capacitances. A set of typical CVs of an AC electrode recorded at different scan rates is displayed in Fig. 17.

In an almost natural development research interest focused on materials with ever larger surface areas, numerous handbooks and collections provide overviews on porous carbons in general [38, 39], and as applied in electrochemistry [40, 41] and in supercapacitors [42, 43]. A larger surface area can be obtained only with smaller particle size, and even more effectively with smaller pore sizes. When the diameter of a pore and in particular its orifice are of the same magnitude as the diameter of an ion (a solvated one in electrolyte solutions and a naked one in molten salts and ionic liquids) the surface area inside this pore may become unreachable for solution and ions and thus useless, consequently a limit of useful porosity and possible surface area is reached. Accordingly with shrinking average pore size lower capacitance values with respect to the mass of carbon are expected despite the larger BETsurface area. As pointed out by Chmiola et al. [44-46] small pores assumed to be inaccessible may, nevertheless, be utilized because of "desolvation" of ions: completely or at least partially desolvated (naked) ions can enter into pores too small to allow entrance of a completely solvated ion. Another property frequently overlooked when chasing

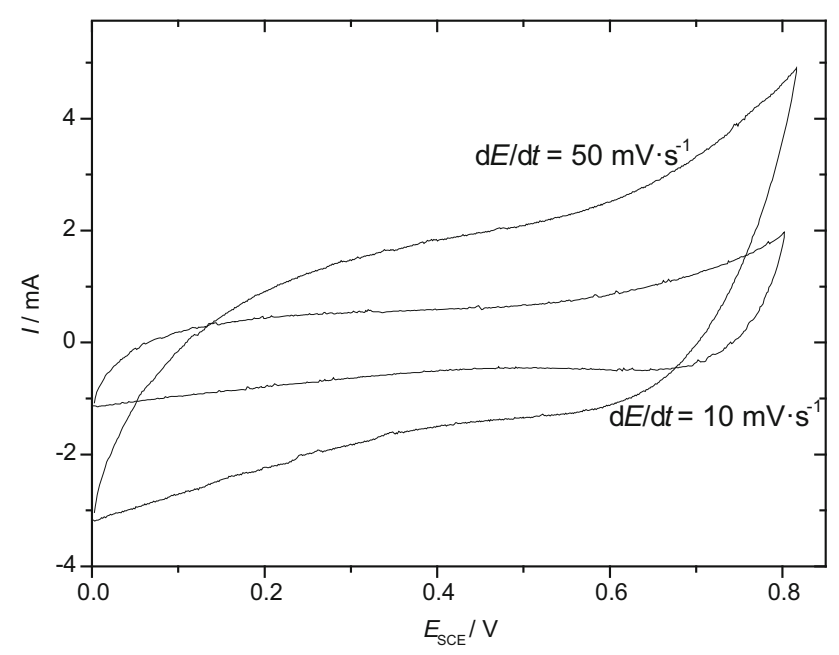

Fig. $17 \mathrm{CV}$ of an electrode of $1.8 \mathrm{mg}$ mass made of $80 \% \mathrm{wt}$. activated carbon, $10 \%$ wt. acetylene black and $10 \%$ wt. PTFE in contact with an aqueous solution of $1 \mathrm{M} \mathrm{LiClO}_{4}$, vs a saturated calomel electrode $E_{\mathrm{SCE}}, \mathrm{d} E / \mathrm{d} t$ as indicated, nitrogen purged for large surface areas is electronic conductance (this applies generally to electrode materials). Low values caused by inherently low conductance of the material or by long and convoluted electronic pathways will limit charge and discharge rates because of the resulting large ESR values (electrical or equivalent series resistance) and thus diminish power density of a device. To some extent electronic properties can be manipulated and improved by nitrogen doping [47] or doping with other hetero-atoms. These challenges and limitations become more serious with thicker electrodes-which in turn cannot be avoided when going from laboratory studies with ultrathin layers of materials to practical electrodes and SCs. Systematic studies of this multidimensional problem have not yet been reported, but the underlying problem becomes more urgent when materials are moving closer to practical application. A first glimpse is provided in Fig. 18.

At smallest loadings-equivalent to a thin film-utilization is highest even at higher scan rates. Nevertheless, a significant amount of active mass remains unused. Assuming a dependence of the utilization on position within the electrode, parts of the electrode at the surface will be utilized preferably. This implies that a loading of $1 / 10$ of the smallest number $(1.45 \mathrm{mg})$ might reach complete utilization. Such thin film is impossible to handle with the doctor-blade technique applied here; it is hardly practical for technical application.

Suitable morphologies, in particular porosities, can be obtained using carbons from natural sources with inherent and thus already established particularly suitable structures, using carbons from polymeric or other synthetic precursors, using templates in the synthesis or using carbon

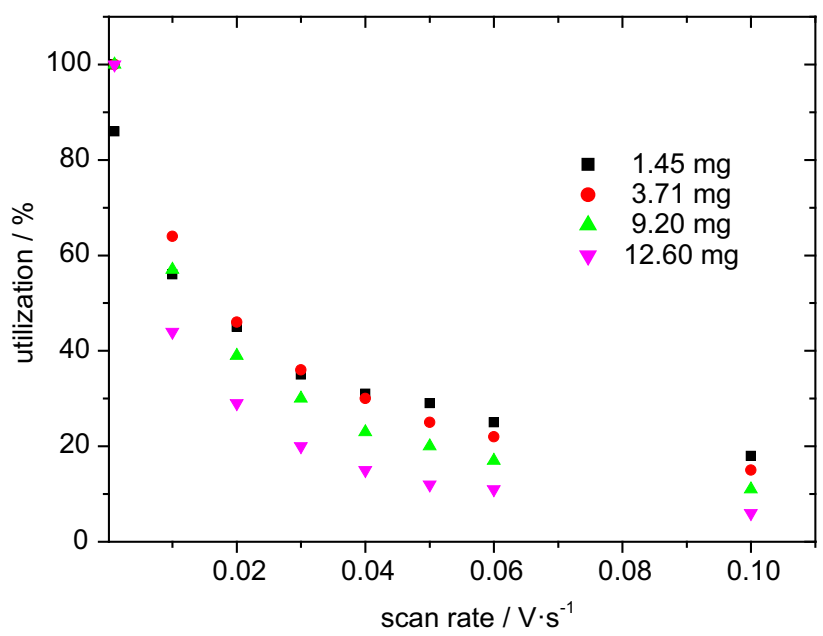

Fig. 18 Utilization of active masses of electrodes with different loadings (see figure) of active mass prepared with $80 \%$ wt. activated carbon, $10 \%$ wt. acetylene black and $10 \%$ wt. PTFE in contact with an aqueous solution of $1 \mathrm{M} \mathrm{LiClO}_{4}$ (unpublished data provided by Sandra Köppen) 
modifications such as nano-fibers, nanotubes, graphenes, etc., [42] aiming at an optimized combination of electronic conductance, large surface in pores just sufficiently accessible (with a rather narrow pore size distribution) and morphology supportive of fast ion transport, and all this at low costs and high life expectancy.

\section{Pseudocapacitive materials}

Pseudocapacitive behavior can be observed with surfaceconfined electrochemical reactions of materials which are insoluble in the employed electrolyte solution. Although well-known examples like formation of hydroxide or oxide coverages on gold or platinum in anodically going electrode potential scans are of no practical importance, the CV of a platinum electrode (Fig. 19) showing formation of a hydroxide/oxide coverage in the positive going scan and subsequent reduction (yielding the visible current peak) in the negative going scan is displayed here for further illustration of the phenomenon and its inherent challenges.

Beyond the obviously inacceptable costs the large difference in electrode potential between oxidation and reduction (this is sometimes called in a slightly imprecise manner irreversibility) would result in large efficiency losses in an SC: the corresponding charging and discharging voltages would differ too much; in electrochemical (and also thermodynamic) terms, the reaction is highly irreversible. Consequently the search has focused on materials showing redox activity with small differences between the redox electrode potentials of the processes operating at high exchange current densities (for an overview of relevant data see [48]). Participating compounds should not undergo significant changes of volume, electronic conductance or any other property relevant for

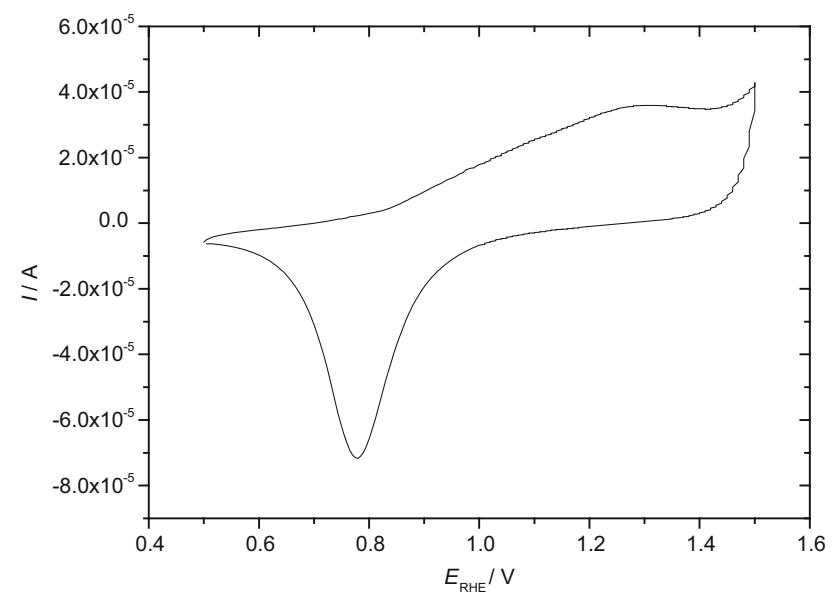

Fig. $19 \mathrm{CV}$ of a polycrystalline platinum electrode in contact with an aqueous solution of $0.05 \mathrm{M} \mathrm{H}_{2} \mathrm{SO}_{4}, \mathrm{~d} E / \mathrm{d} t=0.1 \mathrm{~V} \mathrm{~s}^{-1}$, nitrogen purged operation during these redox processes. In addition, the materials should be cheap and abundantly available, and they should have no negative environmental impact during production and handling. The initially most promising candidate material ruthenium oxide has failed on several of these aspects. The above considerations quite obviously apply not only to metal chalcogenides including oxocompounds but also to electrochemically active polymers.

\section{Metal oxocompounds}

A large number of metal oxides, hydroxides, both binary and multinary ones, have been studied; a review is available [49]. A typical example is shown in Fig. 20, as above weak current waves are superimposed on a large pseudocapacitive current.

The electrode potentials observed with the selected material depend on the potential-determining electrode reaction (for a listing see [48]). Quite obviously a symmetric device with two electrodes made of the same material will provide only a small voltage, thus preferably materials with substantially different electrode potentials should be combined (for a helpful overview see [51]). Particular attention is mostly paid to large BET-surface areas obtained by numerous chemical and electrochemical processes yielding sophisticated nanostructured materials (for an overview and general considerations see [49]). Materials with largest BET-surface area not always yield largest capacitance values. This may be due to fractions of surface area located in pores not accessible for electrolyte ions or simply not wetted. The latter problem is also observed in time-dependent studies as the so-called "activation"; mostly it is just slow soaking of the material or delayed solution penetration into the porous structure. A

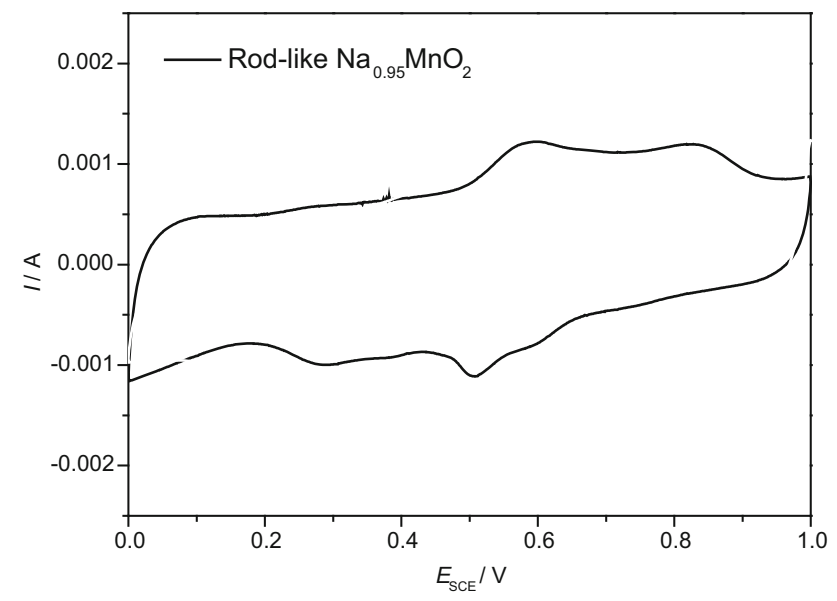

Fig. $20 \mathrm{CV}$ of a rod-like $\mathrm{Na}_{0.95} \mathrm{MnO}_{2}$ electrode in an aqueous solution of $0.5 \mathrm{M} \mathrm{Na}_{2} \mathrm{SO}_{4}$ at $\mathrm{d} E / \mathrm{d} t=5 \mathrm{mV} \mathrm{s}^{-1}$, oxygen is not removed [50] 
further reason for incomplete utilization is related to limited ionic conductance of the electrolyte solution and the electronic conductance of the electrode material. The former causes growing electrolytic resistance to smaller pores in particular with smaller openings, the latter keeps electronic current into very fine structures at low levels getting even lower with finer structures. Detrimental effects of both influences become particularly obvious at higher rates (higher currents): poor conductances (for whatever reason) result in a steep decrease of obtained capacitance with rising current. The term "capacity retention" is applied in describing this phenomenon, but is also used when reporting changes of capacitance as a function of lifetime or cycle number. Both meanings must not be confused. Because of higher Ohmic overpotentials with poorly conducting materials, measured potential limits (stipulated by the onset of unwanted, detrimental side reactions) are reached much earlier, i.e., before fully utilizing available redox sites and surfaces than with better conducting ones. Because the electrode volume is occupied by both materials, the specific conductances of electronically conducting active mass and ionically conducting electrolyte solution are only part of the answer, the actual volume fractions occupied by both phases provide the rest: even a highly conducting solution in a highly porous material with very tortuous pore structure will cause only low effective ionic conductance and thus limit the flow of current, and vice versa. As already discussed above, these limitations and problems become more serious with thicker electrodes. Again a result from preliminary studies provides a first impression (Fig. 21).

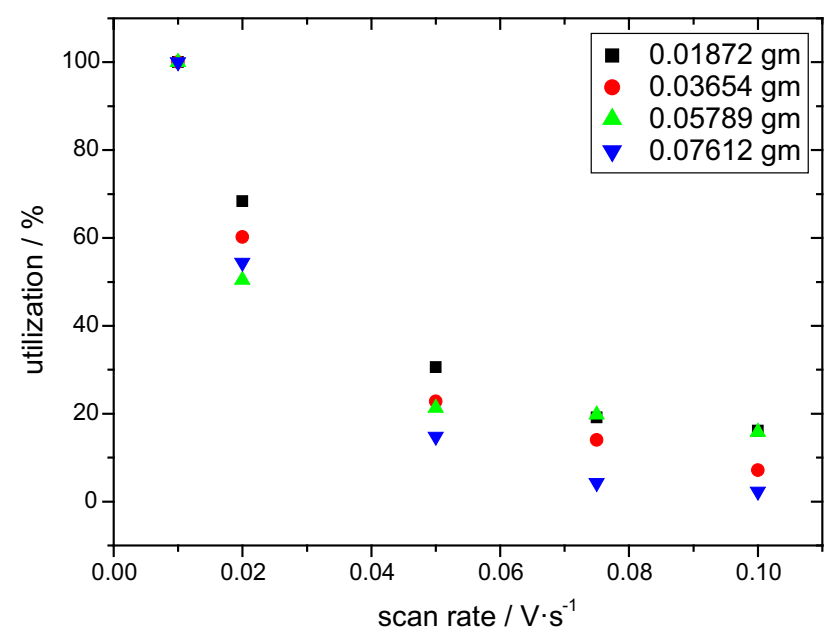

Fig. 21 Utilization of active masses of electrodes with different loadings (see figure) of $\mathrm{LiCoO}_{2}$ prepared by SILAR-method in contact with an aqueous solution of $1 \mathrm{M} \mathrm{LiClO}_{4}$ (Unpublished data provided by Florina Regius)
The rapid drop of actual capacitance with growing scan rate basically very similar to the one observed with a purely capacitive (EDLC) material as shown in Fig. 18 may again have different reasons, poor accessibility and associated unfavorable pore size distribution, insufficient electronic conductance of the active mass and perhaps even hydrophobicity of the material (an aspect unlikely at first glance with a metal oxocompound, but already addressed earlier even with plain metal electrodes [47, 48, 52]).

Thermal stability of nanostructured metal oxides appears to be a challenge so far hardly realized. At first glance, most of the nanostructured materials of whatever chemical composition seem to be perfect candidates for Ostwald ripening, i.e., recrystallization, or agglomeration or other forms of detrimental morphological or structural changes possibly associated with coarsening of the favorable nanostructured form initially present. Because most of the electrochemical processes occur at the surface, substantial shape change beyond the thermodynamically driven processes just addressed is not to be expected, this has been demonstrated in a study of $\mathrm{CuO}$ in neutral electrolyte solution at constant ambient temperature [53]. Unfortunately most authors consider a few thousand charge/discharge cycles enough to call a material stable. This may be appropriate for battery electrodes, and given the slow merger between batteries and SCs the large cycle numbers (also mostly shallow cycles) with capacitors in electronic applications will hardly be the standard in most SC applications, but larger numbers are always preferable.

Morphological changes will be accelerated at elevated temperatures. Almost all reported studies have been performed at room temperature (see also below). A likely application in vehicles will easily provide a substantially different environment. In addition to vibrations, temperatures easily reaching $50{ }^{\circ} \mathrm{C}$ and more are encountered. Joule heating in densely packed electronic circuitry may have similar effects and may cause even higher temperatures. Further stability-relevant details are discussed below. Only future research will show how stable the sophisticated nanostructures will remain.

\section{Intrinsically conducting polymers}

Intrinsically conducting polymers ICPs [54, 55] are frequently electrochemically active showing one or even more redox transformations with electrooxidation(s) associated with respective electroreduction(s). Consequently, they have been studied as active masses for electrochemical conversion and storage devices [56-59]. Because the electrochemically active entities are not discrete ions or molecular species but instead oligomers or conjugated segments of a polymer chain with widely varying numbers of repeat units, redox potentials are not well-defined 
numbers but show distributions of values resulting in wide peaks or waves. Nevertheless, the redox processes determine the operating electrode potential of the ICP. Symmetric devices employing the same material in both electrodes-although suggested-will show only low storage capabilities and low cell voltages as critically reported before [60]. In case of polyaniline PANI thin films show rather well-defined oxidation peaks, but the reduction peak is already much less pronounced (Fig. 22).

The materials and combinations briefly addressed in the previous sections can be put into perspective in Fig. 23.

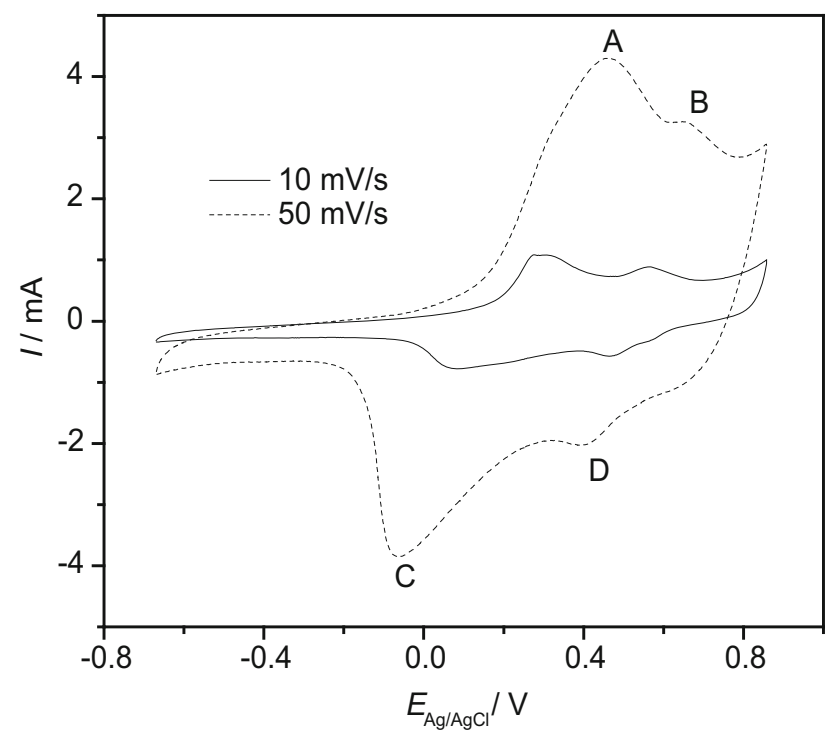

Fig. $22 \mathrm{CVs}$ of PANI at different scan rates in an aqueous $2.0 \mathrm{M} \mathrm{H}_{2} \mathrm{SO}_{4}$ electrolyte solution [61]

\section{The ionically conducting phase in between}

With all active masses the electrolyte solution participates in the proceeding redox reactions. Participation ranges from providing protons or hydroxyl ions required in the redox reactions to supplying ions needed for charge balance in the electrodes. Because electrode reactions initially and predominantly take place at the surface, ingress of anions has frequently been called adsorption without too much attention to the interaction between ion and active material. Beyond this function as sink and source during electrode reactions, the electrolyte solution provides ionic conduction. Thus, requirements for both components and complete phases can be summarized:

- High ionic conductivity

- Compatibility with intended electrode reaction (ion size)

- Chemical stability

- Wide electrochemical window, i.e., large electrode potential difference between anodic and cathodic decomposition

- Cheap and abundantly available

- Environmentally compatible

\section{Stability}

When compared to secondary batteries the superior stability of supercapacitors expressed in terms of charge/discharge cycles is frequently stressed. Even though in both cases a careful and fair comparison would require more precise definition of operating conditions like charge/discharge at

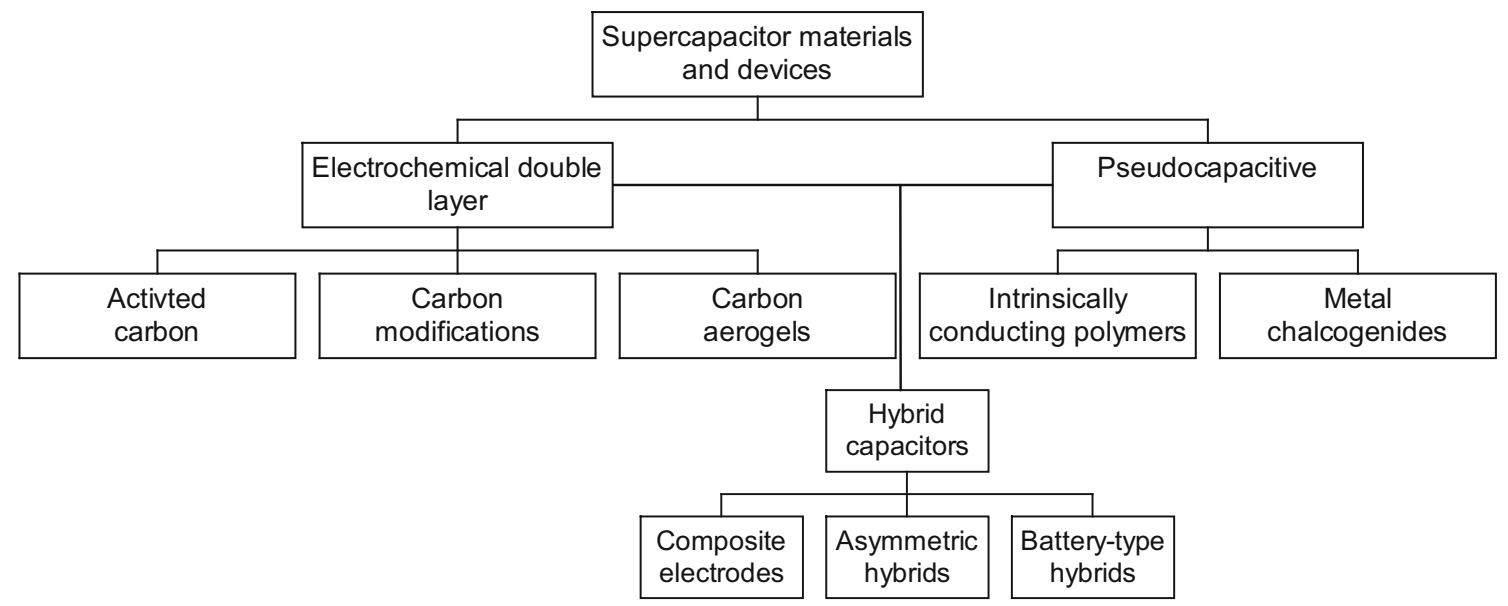

Fig. 23 Taxonomy of supercapacitor materials and devices (based on [32]) 
ambient or extreme temperatures, complete or shallow discharge, etc., it is certainly correct to attribute larger cycling stability to supercapacitors simply because the far-reaching changes associated with chemical transformations inside a battery electrode will not occur in an EDLC device. Supercapacitors employing pseudocapacitive materials already have lost this advantage: redox processes may proceed only or mostly on the very extended exposed surface area of the material being in contact with the electrolyte solution, they are associated, nevertheless, with substantial chemical changes beyond accumulation or redistribution of ions as in EDLCs. Consequently, detrimental changes may happen ranging from agglomeration and loss of active surface area to growth of passivating and possibly insulating surface layers and dissolution of active mass. This will manifest itself as capacity fading, which has been studied only infrequently beyond the popular claim found at the end of most publications that only a few percent fading has been observed after a few thousand cycles. In a typical case of $\mathrm{MnO}_{2}$-based materials [62], the observed fading ranges from 5 to $30 \%$ within thousand cycles depending on current load and binder content. At low binder content and at high current, the transmission resistance (a transmission line model not providing double-layer capacitance information was used as equivalent circuit [63]) deduced from impedance measurements increased indicating growing Ohmic contact resistance between the particles of the redox-active material and the carbon added for improved electric conductance. This was attributed to morphological changes induced by the volume expansion/contraction associated with the redox processes causing mechanical degradation of the electrode. At higher binder content and at lower current densities, the charge transfer resistance related to the electrode reaction grows indicating either a loss of surface area again (no related data were provided), or irreversible changes of the manganese compounds (i.e., transformation from the birnessite phase of $\mathrm{MnO}_{2}$ into $\mathrm{Mn}_{3} \mathrm{O}_{4}$ [64]) or other interfacial changes affecting electrode charge transfer kinetics. Further data range from $5 \%$ per 1000 cycles for NiO$\mathrm{CeO}_{2}$ binary oxide-based electrodes [65] over $1.7 \%$ per 1000 cycles for $\mathrm{LiCr}_{x} \mathrm{Mn}_{2-x} \mathrm{O}_{4}$ [66] to no observable fading with $\mathrm{LiMn}_{2} \mathrm{O}_{4}$ [67].

Already at first glance, pseudocapacitive materials with their highly developed articulate nanostructure seem to be preferred candidates for agglomeration and Ostwald ripening (see above). Although (postmortem) microscope images of active masses of $\mathrm{CuO}$ [53] in aqueous solution of $1 \mathrm{M}$ $\mathrm{Na}_{2} \mathrm{SO}_{4}$ do not show any morphological change after 5000 cycles and thus imply some stability, the behavior of many nanostructured metal oxides at elevated temperatures may pose an entirely different challenge. Temperatures in the range of $50-70{ }^{\circ} \mathrm{C}$ and more can be easily reached under the hood of a car or in a supercapacitor arrangement due to internal Joule heating or environmental contributions.
Extreme, i.e., too negative or positive, electrode potentials applied to pseudocapacitive materials may result in formation of electrochemically less reactive or even inactive materials, gas evolution and morphological changes accompanied by excessive capacity fading as observed with $\mathrm{MnO}_{2}$ [68-70]. Stability of ICPs based on technologically relevant cycle numbers has been reported only infrequently. Pandey and Rastogi reported on a symmetric solid-state device employing PEDOT and a gelled electrolyte without capacity fading after 10,000 cycles [71]. Because of the entirely different mode of operation (for details see above) degradation mechanisms are substantially different, first ideas may be taken from ICP research dealing with their application in smart windows.

Degradation and capacity fading with EDLC devices presently employing preferably carbon-based electrodes will be less severe and caused by different mechanisms. Typical values range from $5.8 \%$ after 8000 cycles [72] to $3.5 \%$ [73] with carbons and less than $1 \%$ [74] or even no fading after 10,000 cycles with nitrogen-doped mesoporous carbons [75]. The beneficial effect of nitrogen doping has been stressed with reasons tentatively associated with electronic effects [47]. No differences between lithium, sodium or potassium ion-based electrolytes in fading were reported [76]. Composites of activated carbon and $\mathrm{Fe}_{2} \mathrm{O}_{3}$ without obvious fading after 1000 cycles [77] or of graphene and $\mathrm{Mn}_{3} \mathrm{O}_{4}$ without significant fading after 1000 cycles [78] may provide options.

Solid electrolytes and organic solvent-based electrolyte solutions may be connected with further fading mechanisms. In the former case with carbon-based electrodes, low fading of about $2 \%$ after 1000 cycles was found [79]; in the latter case, solvent decomposition with film formation on the electrodes was found [80].

\section{Applications}

Initially supercapacitors were used as backup power in small electronic applications instead of secondary batteries, i.e., $\mathrm{NiCd}$ and $\mathrm{NiMH}$ accumulators. Like those systems they are polarized, i.e., they have a negative and positive electrode either by design in asymmetric devices or by application of respective voltages/electrode potentials during manufacturing. Although generally more expensive, they had a huge advantage in being more stable, showing no electrolyte solution leakage, needing no maintenance, no overcharging and rather quick charging. With growing popularity and size initially high prizes came down to values making larger SCs and packs combining several SCs for higher voltage and/or higher current affordable and attractive.

Typical data of commercially a vailable supercapacitors (per 4/2016) 


\begin{tabular}{|c|c|c|c|c|c|c|}
\hline Type/model & Capacitance/F & Cell voltage/V & Energy density $E_{\max } / \mathrm{Wh} \mathrm{kg}^{-1}$ & Power density $P_{\max } / \mathrm{W} \cdot \mathrm{kg}^{-1}$ & $\mathrm{ESR} / \mathrm{m} \Omega$ & Maker \\
\hline HC series & $1 \ldots 150$ & $2.3 \ldots 2.7$ & $0.7 \ldots 4.7$ & $2400 \ldots 7000$ & $14 \ldots 700$ & Maxwell \\
\hline $\mathrm{BC}$ series & $310 \ldots 350$ & 2.7 & $5.2 \ldots 5.9$ & $9500 \ldots 14,000$ & $2.2 \ldots 3.2$ & Maxwell \\
\hline K2 series & $650 \ldots 3400$ & $2.7 \ldots 2.85$ & $4.1 \ldots 7.4$ & $12,000 \ldots 14,000$ & $0.28 \ldots 0.8$ & Maxwell \\
\hline EDLC & $3 \ldots 50$ & 2.7 & $\leq 4.5$ & - & - & Nesscap \\
\hline SkelCap & $2450 \ldots 4500$ & 2.85 & $\leq 14.1$ & - & - & Skeleton \\
\hline Нy-Сар & $1.0 \ldots 500$ & $2.3 \ldots 3.0$ & $\leq 8.7$ & - & - & VinaTecl \\
\hline
\end{tabular}

Thus, the range of application has grown way beyond small devices where SCs simply replaced secondary batteries or provided extra power in combination with fuel cells or less powerful secondary batteries. Beyond these uses, currently SCs are also deployed in large, quickly growing numbers as support or even replacement of lead acid batteries for starting internal combustion engines, for voltage stabilization along electric railway lines making extra supply wires redundant by storing electric energy from braking trains and delivering energy to them when accelerating, for localized energy delivery at high rates for starting electric motors in industrial installations. Even larger modules may be installed in grid applications for power quality management. Reviews reporting further applications and outlining possible future fields of commercialization are available [27, 81]. Many of them are hardly visible, some like the supercap busses running on Renmin road in Shanghai (see Fig. 24) are indicators of the already reached possibilities. According to industry reports, more than 20,000 busses are worldwide operating using supercapacitors in hybrid setups. Compared to batterypowered busses substantial savings both at acquisition (about $40 \%$ less) and during lifetime (the bus is lighter than battery- or motor-driven ones, in addition energy recovery during braking is possible resulting in lifetime fuel cost savings of about 200,000 USD) are observed.

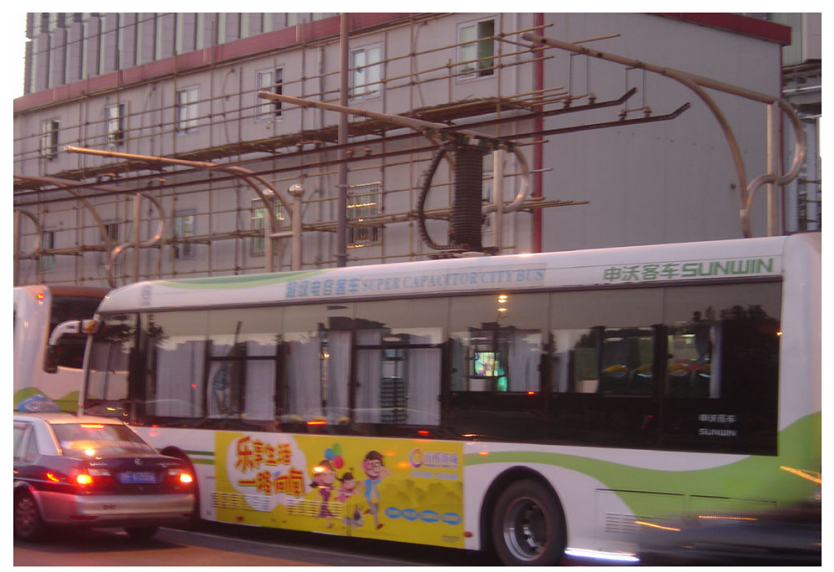

Fig. 24 Supercapacitor bus at the charging station also used as a bus stop
The particular role of supercapacitors in complementing batteries and fuel cells because they can provide high power density mostly lacking in the latter is illustrated in the frequently employed Ragone plot as shown in Fig. 25.

\section{Perspectives}

Currently supercapacitors already successfully employed on a large scale and in a wide range of applications going from small devices supporting memory circuits to large ones in tramway cars, busses and factories are based on EDLC systems. The choice of available pseudocapacitive materials with morphological modifications is growing daily, but presumably because of the lack of sufficiently detailed stability studies industrial applications have not become reality so far. Although the required studies will hardly be spectacular-they seem to be the crucial hurdle this promising technology has to pass. The substantial effects of experimental conditions on reported data have been mentioned and even criticized repeatedly, the effects of testing procedures which will become more important when devices approaching the market have been evaluated [82]. The obvious merger between battery and supercapacitor electrode materials as already pointed out quite

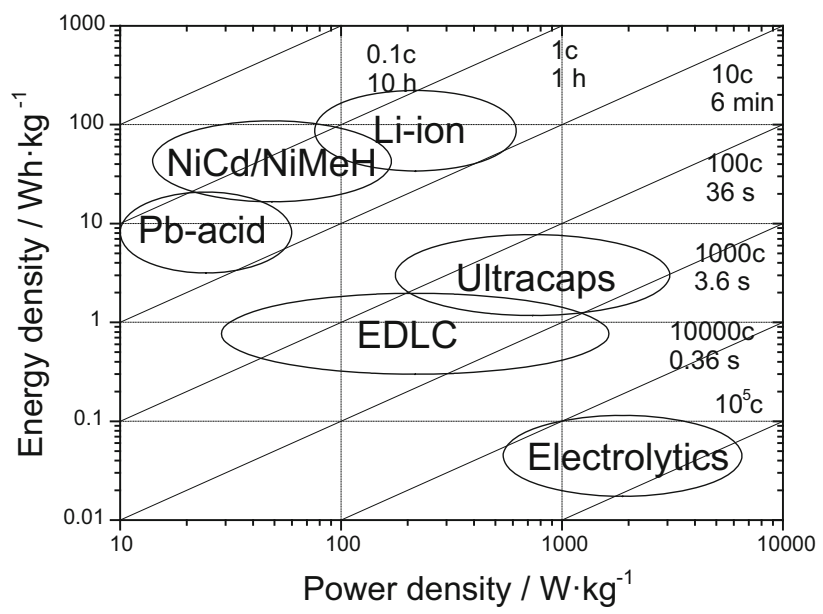

Fig. 25 Ragone plot for selected electrochemical systems, labels: discharge rating in values of $\mathrm{c}$ (rated capacity), theoretical discharge time based on rated capacity 
early [83] will continue, presumably at an accelerated pace (for a recent overview see [84, 85]).

Alternative concepts may at least deserve attention. The use of hydrogen-insertion electrodes based on chemically inactive carbon materials (i.e., no pseudocapacitive redox reaction) utilizes a basically unlimited hydrogen supply from the electrolyte solution [86]. Tian et al. have suggested a device employing dissolved redox couples in two half-cells connected by an ionically conducting membrane [87].

Asymmetric systems combining capacitive (or pseudocapacitive) electrodes with battery electrodes elsewhere found in secondary batteries can help in overcoming inherent limitations of the negative (lead) electrode in a lead acid accumulator [88, 89] by replacing this electrode with a graphite-based capacitive electrode $[90]^{11}$ or can offer new approaches to high-rate systems by combining an aluminum electrode and a graphitic foam electrode in an ionic liquid as electrolyte [91]. Combination with a lithium-ion intercalation negative electrode has been called Li-ion supercapacitor [92]. Recently, sodium-ion capacitors and aluminum-ion capacitors [93, 94] as well as alternative concepts like thermally chargeable SCs have been proposed [95].

Theoretical tools like molecular modeling may increasingly help in understanding and improving behavior of ingredients and electrodes [96].

Acknowledgments Preparation of this report has been supported in various ways by the Alexander von Humboldt-Foundation, Deutscher Akademischer Austauschdienst, Fonds der Chemischen Industrie, Deutsche Forschungsgemeinschaft, National Basic Research Program of China, and Natural Science Foundation of China.

\section{References}

1. Dunn B, Kamath H, Tarascon JM (2011) Science 334:928-935

2. Chen H, Cong TN, Yang W, Tan C, Li Y, Ding Y (2009) Prog Nat Sci 19:291-312

3. Harris C, Meyers JP (2010) Interface 19(3):45-48

4. Miller JR (2009) In: Garche J, Dyer CK, Moseley PT, Ogumi Z, Rand DAJ, Scrosati B (eds) Encyclopedia of electrochemical power sources, vol 1. Elsevier, Amsterdam, pp 587-599

5. Burke A (2009) In: Garche J, Dyer CK, Moseley PT, Ogumi Z, Rand DAJ, Scrosati B (eds) Encyclopedia of electrochemical power sources, vol 1. Elsevier, Amsterdam, pp 685-694

6. Euler KJ (1982) Batterien und Brennstoffzellen. Springer, Berlin

7. Kurzweil P (2009) In: Garche J, Dyer CK, Moseley PT, Ogumi Z, Rand DAJ, Scrosati B (eds) Encyclopedia of electrochemical power sources, vol 3. Elsevier, Amsterdam, pp 596-606

8. Wang G, Zhang L, Zhang J (2012) Chem Soc Rev 41:797-828

9. Kurzweil P (2009) In: Garche J, Dyer CK, Moseley PT, Ogumi Z, Rand DAJ, Scrosati B (eds) Encyclopedia of electrochemical power sources, vol 3. Elsevier, Amsterdam, pp 665-678

$\overline{11}$ This device has been called UltraBattery ${ }^{\circledR}$.
10. von Münch W (1975) Werkstoffe der Elektrotechnik. Teubner, Stuttgart

11. Kurzweil P (2009) In: Garche J, Dyer CK, Moseley PT, Ogumi Z, Rand DAJ, Scrosati B (eds) Encyclopedia of electrochemical power sources, vol. 1. Elsevier, Amsterdam, pp 634-648

12. Becker HI (1957) US-Patent US2800616, 23.07.1957

13. Rightmire RA (1966) US-Patent US3288641, 29.11.1966

14. Kurzweil P (2009) In: Garche J, Dyer CK, Moseley PT, Ogumi Z, Rand DAJ, Scrosati B (eds) Encyclopedia of electrochemical power sources, vol 1. Elsevier, Amsterdam, pp 607-633

15. Boos DL (1970) US-Patent US3536963, 27.10.1970

16. Conway BE, Gileadi E (1962) Trans Faraday Soc 58:2493

17. Conway BE, Pell WG (2003) J Solid State Electrochem 7:637-644

18. Brousse T, Belanger D, Long JW (2015) J Electrochem Soc 162:A5185-A5189

19. Robin MB, Day P (1967) Adv Inorg Radiochem 10:247-422

20. Young MJ, Holder AM, George SM, Musgrave CB (2015) Chem Mater 27:1172-1180

21. Conway BE, Birss V, Wojtowicz J (1997) J Power Sources 66:1-14

22. Beguin F, Frackowiak E (eds) (2013) Supercapacitors. Wiley$\mathrm{VCH}$, Weinheim

23. Burke AF, Miller JR (1996) Electrochemical capacitors. In: Delnick FM, Tomkiewicz M (eds) (Proceedings Volume 95-29). The Electrochemical Society Inc., Pennington, p 281

24. Stoller MD, Ruoff RS (2010) Energy Environm Sci 3:1294-1301

25. Gogotsi Y, Simon P (2011) Science 334:917-918

26. Conway BE (1999) Electrochemical supercapacitors: scientific fundamentals and technological applications. Springer, New York

27. Wang Y, Xia Y (2013) Adv Mater 25:5336-5342

28. Miller JM (2011) Ultracapacitor applications. The Institution of Engineering and Technology, London

29. Yan J, Wang Q, Wei T, Fan Z (2014) Adv Energy Mater 4:1300816

30. Yu A, Chabot V, Zhang J (eds) (2013) Electrochemical supercapacitors for energy storage and delivery-fundamentals and applications. CRC Press, Boca Raton

31. Jayalakshmi M, Balasubramanian K (2008) Int J Electrochem Sci 3:1196-1217

32. Shukla AK, Banerjee A, Ravikumar MK, Jalajakshi A (2012) Electrochim Acta 84:165-173

33. Zhuk A, Denschikov L, Fortov V, Sheindlin A, Wilczynski W (2014) J Appl Electrochem 44:543-550

34. Ma T, Yang H, Lu L (2015) Appl Energ 153:56-62

35. Kan SY, Verwaal M, Broekhuizen H (2006) J Power Sources 162:971-974

36. Adamson AW (1990) Physical chemistry of surfaces. Wiley, New York

37. Thommes M, Kaneko K, Neimark AV, Olivier JP, RodriguezReinoso F, Rouquerol J, Sing KSW (2015) Pure Appl Chem 87:1051-1069

38. Krüger A (2007) Neue Kohlenstoffmaterialien. Teubner-Verlag, Wiesbaden

39. D’Souza F, Kadish KM (eds) (2011) Handbook of carbon nano materials. World Scientific, Singapore

40. Beguin F, Frackowiak E (eds) (2010) Carbons for electrochemical energy storage and conversion systems. CRC Press, Boca Raton

41. Daniel C, Besenhard JO (eds) (2011) Handbook of battery materials. Wiley-VCH, Weinheim

42. Inagaki M, Konno H, Tanaike O (2010) J Power Sources 195:7880-7903

43. Frackowiak E, Abbas Q, Beguin F (2013) J Energ Chem 22:226-240 
44. Chmiola J (2009) PhD-thesis Drexel University, 2009

45. Chmiola J, Yushin G, Gogotsi Y, Portet C, Simon P, Taberna PL (2006) Science 313:1760-1763

46. Chmiola J, Largeot C, Taberna PL, Simon P, Gogotsi Y (2008) Angew Chem Int Ed 47:3392-3395

47. Frackowiak E, Lota G, Machnikowski J, Vix-Guterl C, Beguin F (2006) Electrochim Acta 51:2209-2214

48. Holze R (2007) Landolt-Börnstein: numerical data and functional relationships in science and technology, new series, group IV: physical chemistry, vol 9. In: Martienssen W, Lechner MD (eds) Electrochemistry, subvolume A: electrochemical thermodynamics and kinetics. Springer, Berlin

49. Dubal DP, Holze R (2014) Pure Appl Chem 86:611-632

50. Zhang BH, Liu Y, Chang Z, Yang YQ, Wen ZB, Wu YP, Holze R (2014) J Power Sources 253:98-103

51. Qu Q, Yang S, Feng X (2011) Adv Mater 23:5574-5580

52. Trasatti S (1987) Croat Chem Acta 60:357-370

53. Dubal DP, Gund GS, Holze R, Lokhande CD (2013) J Power Sources 242:687-698

54. Holze R (2001) In: Nalwa HS (ed) Handbook of advanced electronic and photonic materials and devices, vol. 8. Academic Press, San Diego, pp 209-301

55. Holze R (2001) In: Nalwa HS (ed) Advanced functional molecules and polymers, vol. 2. Gordon\&Breach, Amsterdam, pp 171-221

56. Holze R, Wu YP (2014) Electrochim Acta 122:93-107

57. Liangliang T, Chunyang J (2010) Prog Chem 22:1610-1618

58. Ramya R, Sivasubramanian R, Sangaranarayanan MV (2013) Electrochim Acta 101:109-129

59. Zhao C, Zheng W (2015) Front Energ Res 3:23

60. Naoi K, Morita M (2008) Interface 17(1):44-48

61. Reddy Channu VS (2012) Holze R. Iran Polym J 21:457-462

62. Hsieh YC, Lee KT, Lin YP, Wu NL, Donne SW (2008) J Power Sources 177:660-664

63. Fletcher S, Black VJ, Kirkpatrick I (2014) J Solid State Electrochem 18:1377-1387

64. Donne SW (1996) PhD-thesis, University of Newcastle

65. Padmanathan N, Selladurai S (2014) Ionics 20:409-420

66. Wang B, Kang T, Xia N, Wen F, Chen L (2013) Ionics $19: 1527-1533$

67. Wang FX, Xiao SY, Gao XW, Zhu YS, Zhang HP, Wu YP, Holze R (2013) J Power Sources 242:560-565

68. Ataherian F, Wu NL (2011) J Electrochem Soc 158:A422-A427

69. Ataherian F, Wu NL (2011) Electrochem Commun 13:1264-1267

70. Ataherian F, Lee KT, Wu NL (2010) Electrochim Acta 55:7429-7435

71. Pandey GP, Rastogi AC (2012) J Electrochem Soc 159:A1664A1671

72. Wang Q, Cao Q, Wang X, Jing B, Kuang H, Zhou L (2013) J Solid State Electrochem 17:2731-2739
73. Li Z, Zhang L, Amirkhiz BS, Tan X, Xu Z, Wang H, Olsen BC, Holt CMB, Mitlin D (2012) Adv Energy Mater 2:431-437

74. Chen XY, Chen C, Zhang ZJ, Xie DH, Deng X, Liu JW (2013) J Power Sources 230:50-58

75. Zhou DD, Li WY, Dong XL, Wang YG, Wang CX, Xia YY (2013) J Mater Chem A 1:8488-8496

76. Qu QT, Wang B, Yang LC, Shi Y, Tian S, Wu YP (2008) Electrochem Commun 10:1652-1655

77. Wang Y, He P, Zhao X, Lei W, Dong F (2014) J Solid State Electrochem 18:665-672

78. Li L, Seng KH, Chen Z, Liu H, Nevirkovets IP, Guo Z (2013) Electrochim Acta 87:801-808

79. Chiu KF, Su SH (2013) Thin Solid Films 544:144-147

80. Azais P, Duclaux L, Florian P, Massiot D, Lillo-Rodenas MA, Linares-Solano A, Peres JP, Jehoulet C, Beguin F (2007) J Power Sources 171:1046-1053

81. Hall PJ, Mirzaeian M, Fletcher SI, Sillars FB, Rennie AJR, Shitta-Bey GO, Wilson G, Cruden A, Carter R (2010) Energy Environm Sci 3:1238-1251

82. Burke A, Miller M (2010) Electrochim Acta 55:7538-7548

83. Conway BE (1991) J Electrochem Soc 138:1539-1548

84. Cericola D, Kötz R (2012) Electrochim Acta 72:1-17

85. Dubal DP, Ayyad O, Ruiz V, Gomez-Romero P (2015) Chem Soc Rev 44:1777-1790

86. Qu D, Smith P, Gourdin G, Jiang T, Tran T (2012) Chem Eur J 18:3141-3143

87. Tian ZW, Dong QF, Zheng MS, Lin ZG (2009) US20090190286, 30.07.2009

88. Lam L.T, Furukawa J (2009) in: Garche J, Dyer CK, Moseley PT, Ogumi Z, Rand DAJ, Scrosati B (eds) Encyclopedia of Electrochemical Power Sources Vol. 4, Elsevier, Amsterdam, p. 755-763

89. Kurzweil P (2009) In: Garche J, Dyer CK, Moseley PT, Ogumi Z, Rand DAJ, Scrosati B (eds) Encyclopedia of electrochemical power sources, vol. 1. Elsevier, Amsterdam, pp 658-664

90. Banerjee A, Ramasesha SK, Shukla AK (2014) Electrochem Energ Technol 1:10-16

91. Lin MC, Gong M, Lu B, Wu Y, Wang DY, Guan M, Angell M, Chen C, Yang J, Hwang BJ, Dai H (2015) Nature 520:325-328

92. Li J, Xu M, Li X, Yuan C (2012) Adv Mat Res 482-484:448-451

93. Wang FX, Wang XW, Chang Z, Wu XW, Liu X, Fu LJ, Zhu YS, Wu YP, Huang W (2015) Adv Mater 27:6962-6968

94. Wang FX, Wang XW, Liu ZC, Yuan XH, Wu XW, Fu LJ, Zhu YS, Wu YP (2016) to be published

95. Lim H, Zhao C, Qiao Y (2014) Phys Chem Chem Phys $16: 12728-12730$

96. Burt R, Birkett G, Zhao XS (2014) Phys Chem Chem Phys 16:6519-6538 